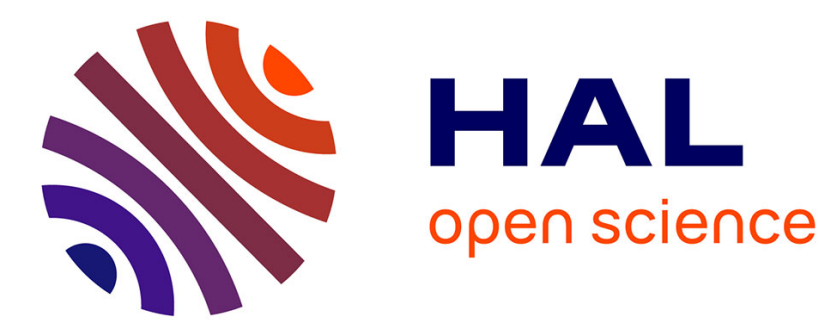

\title{
Falling jet of dry granular material in water
}

Guillaume Saingier, Alban Sauret, Pierre Jop

\section{To cite this version:}

Guillaume Saingier, Alban Sauret, Pierre Jop. Falling jet of dry granular material in water. Journal of Fluid Mechanics, 2021, 10.1017/jfm.2021.131 . hal-03166692v2

\section{HAL Id: hal-03166692 \\ https://hal.science/hal-03166692v2}

Submitted on 2 Apr 2021

HAL is a multi-disciplinary open access archive for the deposit and dissemination of scientific research documents, whether they are published or not. The documents may come from teaching and research institutions in France or abroad, or from public or private research centers.
L'archive ouverte pluridisciplinaire HAL, est destinée au dépôt et à la diffusion de documents scientifiques de niveau recherche, publiés ou non, émanant des établissements d'enseignement et de recherche français ou étrangers, des laboratoires publics ou privés. 


\title{
Falling jet of dry granular material in water
}

\author{
G. Saingier ${ }^{1}$, A. Sauret ${ }^{2}$, and P. Jop ${ }^{1} \dagger$ \\ ${ }^{1}$ Surface du Verre et Interfaces, UMR 125, CNRS/Saint-Gobain, 39, quai Lucien Lefranc, \\ F-93303 Aubervilliers, Cedex France \\ ${ }^{2}$ Department of Mechanical Engineering, University of California, Santa Barbara, CA, USA
}

(Received xx; revised xx; accepted xx)

Modeling the flow of dry granular materials entering water is crucial to optimize blending processes in the industry or for natural hazard assessment when describing the tsunami waves induced by landslides. In this study, we experimentally investigate the case of a jet of grains entering from the air into a water bath. After an initial transient state, a stationary impregnation front appears between the dry and the wet grains. The wet grains are then dispersed in the liquid. To describe this dry to wet transition, we focus on the first step of the process when the liquid invades the dense granular medium. In this regime, the granular jet is modeled as a translating porous material, and we systematically characterize the impregnation process using a combination of experiments, analytical modeling, and numerical tools. We then compare this approach to the situation of a confined granular jet entering into a water bath. Our approach is a first step toward describing the interplay between dry grains and a liquid and the resulting dispersion of particles.

Key words: porous media, granular media, particle/fluid flow

\section{Introduction}

Blending grains with a liquid is an essential step in many industrial processes, for instance in the food industry (Forny et al. 2011) and in civil engineering for the preparation of building materials (Cazacliu \& Roquet 2009; Collet et al. 2010). The mixing step often involves initially pouring granular materials into a liquid bath so that the particles are dispersed in the interstitial fluid. At a larger scale, the entry of grains into water occurs during the collapse of a cliff edge at the seaside. In this situation, tsunami waves can be generated by the entry of the granular mass into the ocean, which leads to a significant hazard for the population. This phenomenon has been the subject of experimental works to quantify the amplitude of the waves resulting from these events (Fritz et al. 2003; Heller et al. 2008; Viroulet et al. 2013, 2014). Macroscopic theoretical models describing the energy transfer between the grains and the liquid have been proposed to predict the amplitude of the wave generated (Zitti et al. 2016; Mulligan \& Take 2017). Nevertheless, understanding of the interplay between the grains and the liquid is essential to correctly describe the transition from dry to wet grains during the immersion of a dense granular material. This interaction is also important to predict the liquid and sediment transports in complex granular systems, for instance during the drainage of water from the basements after heavy rains (Horton 1945; Kirchner et al.

$\dagger$ Email address for correspondence: Pierre.Jop@saint-gobain.com 

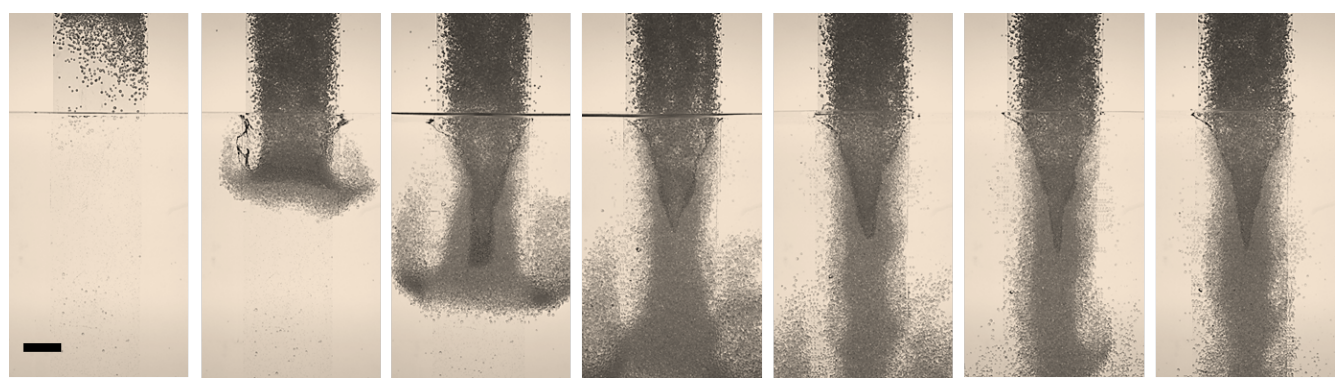

Figure 1. Time series of a free semi-2D granular jet penetrating into a water bath. Particles are spherical glass beads of diameters $d_{g}=1050 \pm 250 \mu \mathrm{m}$. The jet of width $5 \mathrm{~cm}$ is confined between two PMMA plates (at the front and the rear). Each picture are separated by $0.25 \mathrm{~s}$. Scale bar is $2 \mathrm{~mm}$.

2000; Guérin et al. 2014), which also impact the stability of soils subjected to extreme conditions (Herminghaus 2005).

The interplay between a granular material and a liquid has been extensively studied in static configurations where the granular material behaves as a porous medium in which the liquid flows. At low Reynolds number, the flow dynamics is captured by the Darcy's law, relating the fluid velocity to the pressure gradient applied to the liquid (Bear 1988). For larger Reynolds numbers, inertial effects induce a nonlinear dependence of the pressure gradient with the velocity. Different inertial corrections have been reported in the literature (Bear 1988). Among them, the Forchheimer's law has successfully been used to capture various situations of flow in porous media (Bear 1988). These approaches are used to describe, for instance, capillary flows in porous media in different geometries (Lucas 1918; Washburn 1921; Hyväluoma et al. 2006; Xiao et al. 2012; Benner \& Petsev 2013), and various layered porous systems (Reyssat et al. 2009; Mensire et al. 2016). Gravity flows in porous media, such as during the drainage in aquifers, have also been successfully described using these models (Lyle et al. 2005; Vella \& Huppert 2006; Guérin et al. 2014). Nevertheless, the dynamics situation of a porous media plunging from the air into a water bath remains elusive.

In this work, we investigate the entry of a dry granular jet in a liquid bath. The granular jet configuration is well-known in the case of an impact on a solid surface (Cheng et al. 2007; Müller et al. 2014). Conversely, the interaction with a soft or a liquid surface remains a subject of interrogation. When the granular material penetrates the liquid bath, it tends to fragment by interacting with the fluid (González Gutiérrez et al. 2014; Cervantes-Álvarez et al. 2020). Once immersed, the flow of grains in an interstitial liquid is governed by the viscous displacement of the particle in the fluid (Courrech du Pont et al. 2003; Doppler et al. 2007; Topin et al. 2012; Bougouin et al. 2017).

Modeling a dry granular jet entering into water is a complex problem since the structure of the jet evolves as the grains enter the fluid. Contrary to a jet of an immiscible liquid into water, the granular jet impregnates when the particles that compose it are hydrophilic, leading to a complex multiphase situation. Figure 1 shows a time series of a jet of dry grains entering into a quiescent water bath. In the configuration considered here, when the jet crosses the liquid surface, it remains compact and deforms the free-surface. The jet begins to impregnate and has a dry part located inside the jet (dark gray) surrounded by an impregnated part (light gray). After a short transient, a stationary V-shaped impregnation front appears. The grains only disperse after being entirely wetted by the water. This observation suggests modeling at first order the initial behavior of a dense granular jet as a porous medium translating into a liquid bath. This situation is 


$\begin{array}{lccccccc}\text { Syst. } & d_{g}(\mu \mathrm{m}) & k\left(10^{-10} \mathrm{~m}^{2}\right) & k_{1}\left(10^{-10} \mathrm{~m}^{2}\right) & k_{2}\left(10^{-10} \mathrm{~m}^{2}\right) & \beta_{2}\left(10^{5} \mathrm{~m}^{-1}\right) & \theta_{c}\left(^{\circ}\right) & \phi \\ \text { W1 } & 300-380 & 0.7 & 0.8 & 0.8 & 3.1 & 42 \pm 5 & 0.59 \\ \text { W2 } & 400-470 & 1.0 & 1.0 & 1.3 & 1.4 & 33 \pm 5 & 0.61 \\ \text { W3 } & 800-1300 & 10 & 11.6 & 12.1 & 0.1 & 45 \pm 5 & 0.62 \\ \text { N1 } & 140-320 & 1.1 & 1.1 & - & - & 76 \pm 5 & - \\ \text { N2 } & 280-420 & 5.5 & 5.5 & - & - & 72 \pm 5 & -\end{array}$

TABle 1. Physical properties of the grains composing the model porous media.

reminiscent of the coating processes used to coat textiles or surfaces with a liquid coating by dragging them into a bath (Quéré 1999; Clarke 2002; Seiwert et al. 2011). However, our configuration differs by the complexity of the surface exposed to the liquid, namely a porous material that can be impregnated by the liquid. Furthermore, in the case of a porous material, the isotropy of the medium does not constrain an orientation of the fluid front, contrary to the hairy surfaces studied recently by Nasto et al. (2016).

In this work, we experimentally investigate the impregnation of a porous material translated into a liquid reservoir. The experimental observations are compared to analytical models and numerical results. The experimental setup is described in section 2. The transient impregnation regime is reported in section 3 and modeled by a $1 \mathrm{D}$ impregnation dynamics. In section 4 , we focus on the stationary regime characterized by a stationary impregnation. This stable profile is then partially modeled using Darcy's and Forchheimer's models in section 5. In section 6, a numerical method is developed to model the shape of the impregnation front fully. Finally, a discussion is proposed in section 7 to compare the results obtained with a porous medium to those obtained with a dense granular jet.

\section{Experimental methods}

\subsection{Experimental set-up}

The experimental setup, shown in figure 2(a), consists of a linear motor stage translating a porous material at a constant velocity $V_{0}$ into a water reservoir of large dimensions. The porous medium is formed by packing spherical glass beads into a transparent cell of length $40 \mathrm{~cm}$, of variable width $W=5,10$, and $20 \mathrm{~cm}$ and of thickness $12 \mathrm{~mm}$ so that the configuration can be considered as bidimensional, as shown in figure 2(b). The side and bottom walls of the cell are permeable thanks to a metal wire mesh of opening $250 \mu \mathrm{m}$ [visible in figure 2(a)]. The small thickness of the cell allows us to visualize the dry and wet grains in dark and light, respectively, using the contrast of absorption of the light emitted by a LED panel placed behind the setup [figure 2(c)]. It also allows us to track the impregnation front separating the dry and wet regions.

The porous medium is translated vertically into the water bath at a constant speed $V_{0}$ varying between $1 \mathrm{~mm} / \mathrm{s}$ and $150 \mathrm{~mm} / \mathrm{s}$. The impregnation dynamics is recorded at 30 frames per second (Nikon D-7100 with an F30 lens) and then analyzed by image processing to extract the shape of the impregnation front and its time-evolution during the experiment.

\subsection{Characterization of porous media}

The porous medium is prepared by filling the transparent cell with glass beads. Different systems of grains were used to tune the pore size and the wettability of the 


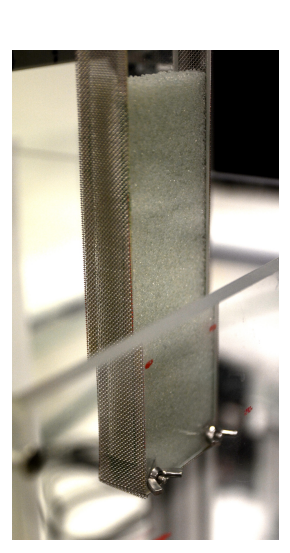

(a)

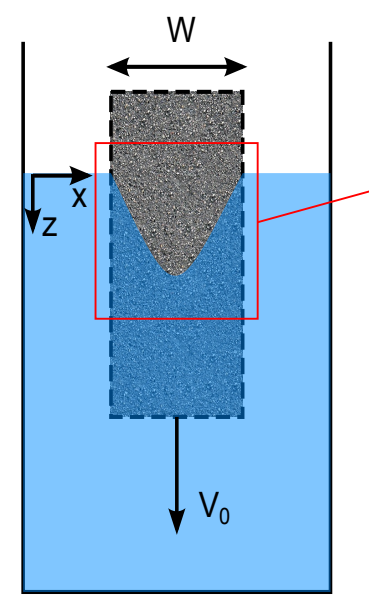

(b)

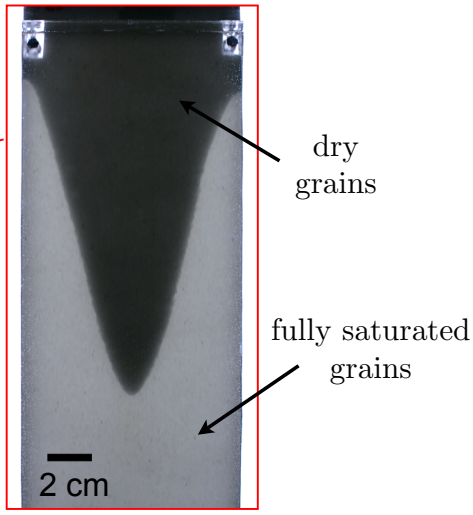

(c)

Figure 2. (a) Photo of the porous medium made of grains (system W3). The front and rear sides are made of PMMA, and the lateral sides and the bottom of the cell consist of a metal wire mesh of opening $250 \mu \mathrm{m}$. (b) Schematic of the experimental setup. A vertical linear motor imposes the speed of translation $V_{0}$. (c) Picture of the stationary impregnation front during the translation of the porous medium into the water bath. The system is illuminated by a LED panel placed behind the cell. The light part is fully saturated in water whereas the dark part is dry.

porous medium. We performed the experiments with the systems W1, W2, W3, where W stands for wetting, and the systems N1 and N2, where N stands for non-wetting thanks to coated glass beads (Sigmund Lindner). The coating changes the surface properties of the beads by increasing the contact angle with the liquid. The beads are sieved to refine the size distributions which are summarized in table 1 (see also Appendix A). For each system, the glass beads are cleaned with soap and thoroughly rinsed with de-ionized water to remove any dust on the grains.

The contact angle between the grain and the water is measured by trapping a single bead at the surface of a pending drop of liquid (Timounay et al. 2015). For each experiment, the granular packing is prepared by following a protocol detailed in Appendix A. Several taps are given to compact the granular packing until reaching a constant volume fraction of $\phi \simeq 0.62$. The compacity $\phi$ and the porosity $\epsilon$ of the granular packing are estimated by measuring the mass and the volume occupied by the grains (see Appendix A). The permeability of the porous media, $k$, is measured by an experiment of filtration through the porous medium following the procedure presented by Chopin \& Kudrolli (2011). This measurement is performed at low Reynolds number, in the range of velocities compatible with the Darcy's law. The measured permeability is noted $k_{1}$ in table 1 . Another set of measurements of the permeability is performed at larger Reynolds numbers to determine the Forchheimer's coefficient $\beta_{2}$ required in the DarcyForchheimer's equation used in section 5. Furthermore, a third value of the permeability, denoted $k$, is used to fit the theoretical prediction to the experimental data. Only the values $k$ and $\beta_{2}$, are used in this paper. The discrepancies between each measurement of the permeability are relatively small and remain less than $20 \%$ for all experimental configurations. The small difference can be explained by experimental artifacts brought by the glass tube used for the flow measurements through the packed beads. The physical characteristics of all model porous media used in this study are summarized in table 1 , 


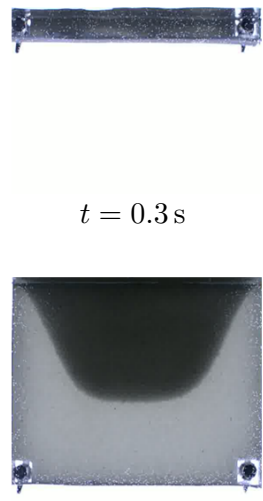

$t=2.6 \mathrm{~s}$

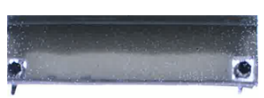

$t=0.8 \mathrm{~s}$

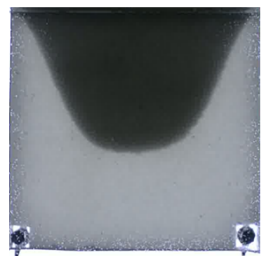

$t=3.1 \mathrm{~s}$

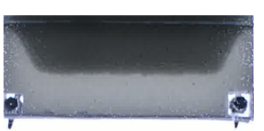

$t=1.2 \mathrm{~s}$

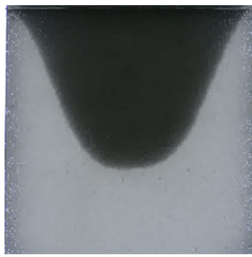

$t=3.6 \mathrm{~s}$

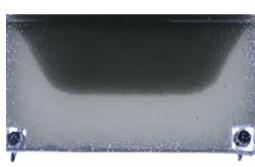

$t=1.7 \mathrm{~s}$

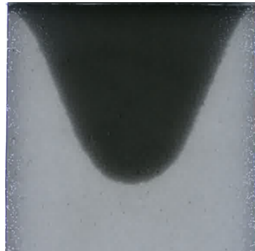

$t=4.0 \mathrm{~s}$

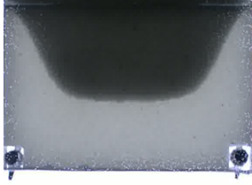

$t=2.2 \mathrm{~s}$

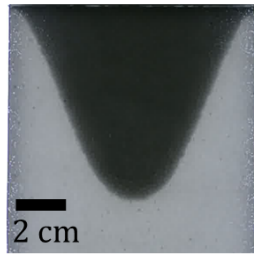

$t=4.5 \mathrm{~s}$

FiguRE 3 . Time series during the transient phase of a porous medium of width $W=10 \mathrm{~cm}$ composed of the system W2 plunging into water at a speed $V_{0}=30 \mathrm{~mm} / \mathrm{s}$.

and all the experimental methods used to characterize the properties of the beads are detailed in Appendix A.

\subsection{Phenomenology}

An example of time-series extracted from an experiment is shown in figure 3 . This experiment is performed with a porous medium made of glass beads from the system W1 in a $5 \mathrm{~cm}$-wide cell translating into a water bath at a constant velocity $V_{0}=30 \mathrm{~mm} / \mathrm{s}$. When the porous medium plunges into the water, air is entrained within the porosity of the material, and liquid penetrates laterally and vertically into the pores, which leads to a V-shaped impregnating front visible in the last image. This front separates the part of the porous medium invaded by the liquid (the wet grains, light region in figure 3) to the dry part of the sample containing only air in the porosity (visible in dark). The dynamical impregnation is characterized by a transient phase for $t<\tau$, where $\tau$ is the time to reach a stationary impregnation profile. The transient phase is followed by a stationary regime for $t>\tau$. During the transient phase, the impregnation front evolves through different shapes until reaching a steady $\mathrm{V}$-shape in the stationary regime.

\section{Transient regime}

We first focus in this section on the transient regime observed for $t<\tau$. The transient regime is characterized by the continuous evolution of the impregnation front until reaching the stationary $\mathrm{V}$-shape profile, which will be characterized in section 4 .

\subsection{Dynamics of impregnation}

We study the transient regime by tracking the temporal evolution of the impregnation front. The shape of the impregnation front is reported at different times in figure 4(a) for an experiment performed with a porous medium of width $W=10 \mathrm{~cm}$ composed of grains from the system $\mathrm{W} 2$ and plunged in the water bath at a constant velocity $V_{0}$ $=30 \mathrm{~mm} / \mathrm{s}$. The impregnation front evolves continuously from an initial flat profile to a V-shape profile by keeping a constant slope on the side. The temporal evolution of the impregnation is characterized by measuring the vertical length of air entrained in the bath, noted $\ell_{d r y}(t)$ [see figure $4(\mathrm{a})$ ]. The slope of the impregnation front remains constant 


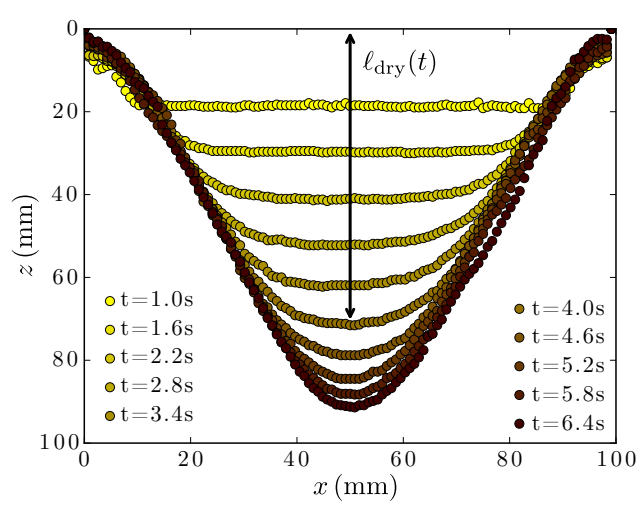

(a)

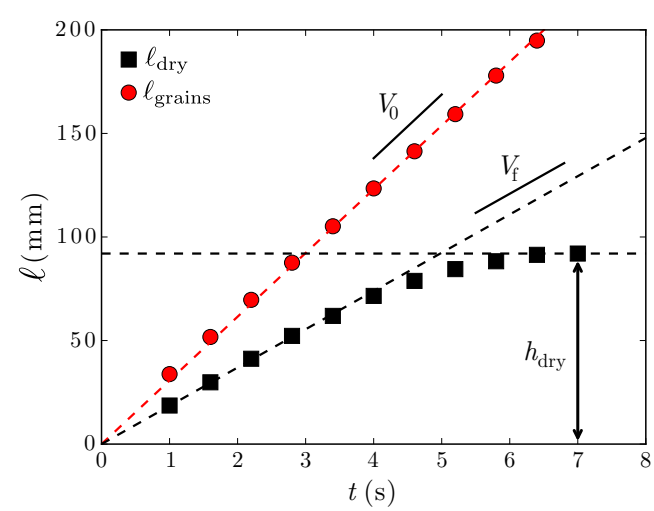

(b)

Figure 4. (a) Evolution of the impregnation front during the transient regime. The data are extracted from the experiment shown in figure 3. The air-water interface is located at $z=0$. (b) Evolution of the maximum length of air $\ell_{\mathrm{dry}}(t)$ entrained under the liquid surface. The red circles corresponds to the position of the bottom of the porous medium that is translated at a constant velocity $V_{0}$ under the surface.

during all the process and will be characterized in the next section. The time evolution of $\ell_{d r y}(t)$ is presented in figure $4(\mathrm{~b})$. The length $\ell_{d r y}(t)$ increases linearly with a velocity $V_{f}$ before saturating to a constant value, $\ell_{d r y}(t \rightarrow \infty)=h_{d r y}$, which corresponds to the maximum length of air entrained by the porous material when the stationary regime is reached.

Using $h_{d r y}$ and $\tau=h_{d r y} / V_{f}$ as characteristic length scale and time scale, respectively, we can rescale the evolution of $\ell_{d r y}(t)$ for all the experiments performed with the different model porous media. The results are reported in figure 5 and show that all the rescaled data collapse on an empirical law with a single fitting parameter:

$$
\frac{\ell_{d r y}}{h_{d r y}}=f\left(\frac{t}{\tau}\right) \quad \text { with } \quad f(\xi)=\left(1-e^{-\xi^{n}}\right)^{1 / n} \quad \text { and } \quad n \simeq 4.5 .
$$

The impregnation by the fluid from the bottom wall of the porous medium governs the evolution of $\ell_{d r y}$ during the transient regime. In parallel, the liquid invades the porous medium from the lateral walls. The resulting impregnation profile exhibits a constant slope resulting from the balance between the liquid impregnation and the translation of the porous medium. The stationary regime is reached when the two impregnation fronts from each side meet to form the stationary $\mathrm{V}$-shape profile. Before that, the vertical impregnation length $\ell_{d r y}(t)$ is limited by the vertical 1D flows coming from the bottom of the cell. Therefore, the transient regime and the linear time evolution of $\ell_{d r y}(t)$ before the saturation can be modeled by considering the vertical impregnation in a uniform $1 \mathrm{D}$ porous medium translating into a liquid bath.

\subsection{D-Imbibition model}

We consider a 1D porous medium with impermeable sidewalls and a porous bottom wall translated at a constant speed $V_{0}$ into a liquid bath (see figure 6 ). The porous material is characterized by its permeability $k$, its compacity $\phi$, the average pore diameter $\bar{d}_{g}$ and the contact angle $\theta_{c}$ of the three-phase contact line on the glass beads. The capillary pressure drop at the impregnation front, $p_{c}$, is given by (Reyssat et al. 2009; Xiao et al. 


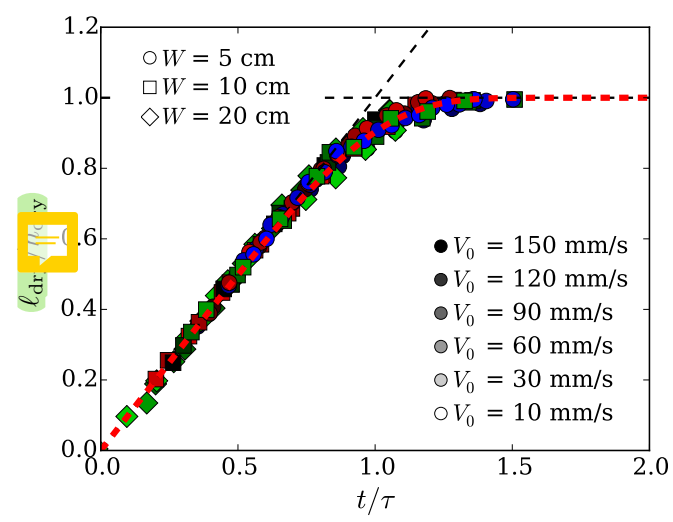

FiguRE 5. Rescaled length of air entrained under the free surface $\ell_{d r y} / h_{d r y}$ as a function of the dimensionless time $t / \tau$. The figure summarizes the results of 30 sets of parameters varying the size of the grains, the width of the cell $W$ and the plunging speeds $V_{0}$. Experiments with beads of systems W1, W2 and W3 are plotted in blue, red and green, respectively. The red dashed line is the empirical fit given by equation (3.1).

2012):

$$
p_{c}=\frac{4 \gamma \cos \theta_{c}}{\bar{d}_{g}},
$$

where $\gamma \simeq 70 \mathrm{mN} \cdot \mathrm{m}^{-1}$ is the air-water surface tension. We also define the Reynolds number associated to the fluid displacement in the porous material as

$$
R e_{p}=\frac{\rho u \bar{d}_{g}}{\eta},
$$

where $\rho$ is the density of the fluid, $\eta$ the viscosity and $u$ its characteristic velocity in the porous medium. In the transient regime studied here, an order of magnitude of the velocity $u$ is about $10 \mathrm{~mm} / \mathrm{s}$, resulting in a Reynolds number $R e_{p}$ in the range 1-10, depending on the grain sizes. In this range of Reynolds numbers, the flow dynamics into the porous medium is modeled by the Darcy's equation, which relates the average liquid velocity $\boldsymbol{u}_{\text {Darcy }}$ to the pressure gradient $\boldsymbol{\nabla} p$ (Mensire et al. 2016):

$$
\boldsymbol{u}_{\text {Darcy }}=(1-\phi) \boldsymbol{u}_{p}=-\frac{k}{\eta}(\nabla p-\rho \boldsymbol{g})
$$

where $\boldsymbol{u}_{p}$ is the mean velocity of the liquid into the porous medium, and $\boldsymbol{g}$ is the gravitational acceleration. In this configuration, the liquid flow is driven both by the drop of capillary pressure at the interface and the hydrostatic pressure.

We note $\ell_{0}$ the position of the bottom of the porous medium such that $\ell_{0}$ is the total length of the material immersed under the liquid surface. This length can be decomposed as the sum of the wet part $\ell_{w e t}$ and the dry part $\ell_{d r y}$, as shown in figure 6 . The porous material is translated at a constant speed $V_{0}$, so that $\ell_{0}=V_{0} t=\ell_{\text {dry }}+\ell_{\text {wet }}$.

The Darcy's equation (3.4) in one dimension, along the $z$ axis, is then

$$
\frac{\mathrm{d} \ell_{w e t}}{\mathrm{~d} t}=\frac{k}{(1-\phi) \eta}\left(\frac{p_{c}+\rho g \ell_{0}}{\ell_{w e t}}-\rho g\right),
$$

where the fluid velocity at the pore scale is associated to the velocity of the impregnation front $u_{p}=\mathrm{d} \ell_{d r y} / \mathrm{d} t$. We introduce $V^{\star}$ as the characteristic impregnation velocity 


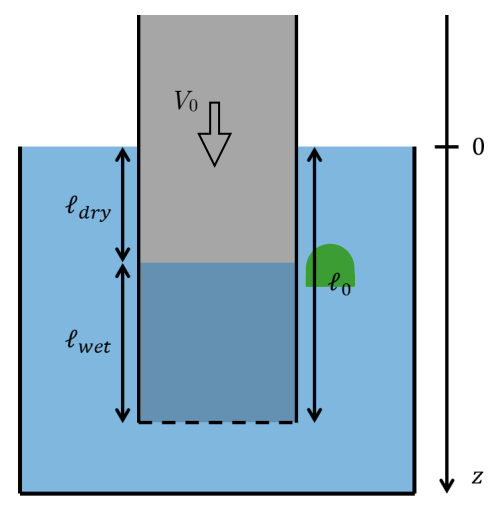

FIGURE 6 . Schematic of the impregnation of a $1 \mathrm{D}$ porous medium plunging in water at a constant velocity $V_{0}$ along the $z$ axis. The total length of the porous medium immersed in water is denoted $\ell_{0}, \ell_{\mathrm{dry}}$ and $\ell_{\text {wet }}$ corresponds to the dry and wet length under the surface, respectively.

associated to the gravity-driven flow:

$$
V^{\star}=\frac{k \rho g}{(1-\phi) \eta} .
$$

We also introduce the dimensionless length $L_{w e t}=\ell_{w e t} / h_{\infty}$ and dimensionless time $T=t V^{\star} / h_{\infty}$, where $h_{\infty}$ is the Jurin's height defined by the balance of the capillary forces and the liquid weight such that $h_{\infty}=p_{c} / \rho g$. The Eq. (3.5) then becomes

$$
\frac{\mathrm{d} L_{w e t}}{\mathrm{~d} T}=\frac{1+\left(V_{0} / V^{\star}\right) T}{L_{w e t}}-1 .
$$

This equation can be solved analytically, as detailed in Appendix B. The theoretical impregnation dynamics are computed for different values of $V_{0} / V^{\star}$ and reported in figure $7(\mathrm{a})$. For $V_{0} / V^{\star}=0$, the dynamics reduces to the well-known Lucas-Washburn's equation for the vertical impregnation of a liquid in a static porous medium under gravity (Washburn 1921; Lucas 1918). The fluid displacement in the porous medium is controlled by the capillary imbibition and follows a diffusion dynamics in $t^{1 / 2}$ before saturating under the effect of gravity at the Jurin's height $h_{\infty}$ (Delker et al. 1996; Lago \& Araujo 2001). For moderate values of $V_{0} / V^{\star}$, a capillary regime is observed at short time, followed by a pressure-driven regime exhibiting a constant impregnation velocity $V_{\text {wet }}$ as shown in figure $7(\mathrm{a})$. These front velocities are reported in figure $7(\mathrm{~b})$ as a function on the dimensionless plunging speed $V_{0} / V^{\star}$. We can derive an analytical expression of the impregnation front velocity $V_{\text {wet }}$ by simplifying the Eq. (3.7) at long time, i.e. for $\left(V_{0} / V^{\star}\right) T \gg 1$. In this regime, the capillary term can be neglected with respect to the hydrostatic term and the equaqtion becomes:

$$
\frac{\mathrm{d} L_{w e t}}{\mathrm{~d} T}=\left(\frac{V_{0}}{V^{\star}}\right) \frac{T}{L_{w e t}}-1 .
$$

This equation has a solution of the form $L_{\text {wet }}=\nu T$ where $\nu$ is a constant (Mullins \& Braddock 2012). This kind of solution is consistent with the experimental observations, where the front velocity is constant at long time in the transient regime before saturating when the stationary state is reached. Injecting the ansatz $L_{w e t}=\nu T$ in equation (3.8), we obtain

$$
\nu^{ \pm}=-\frac{1}{2} \pm \frac{1}{2} \sqrt{1+4\left(\frac{V_{0}}{V^{\star}}\right)},
$$




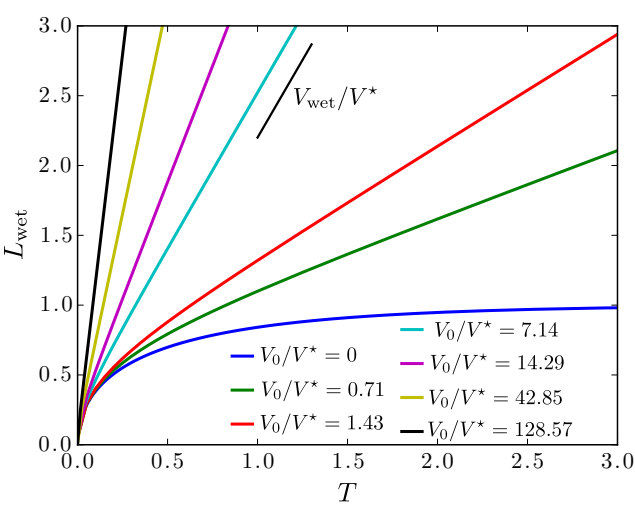

(a)

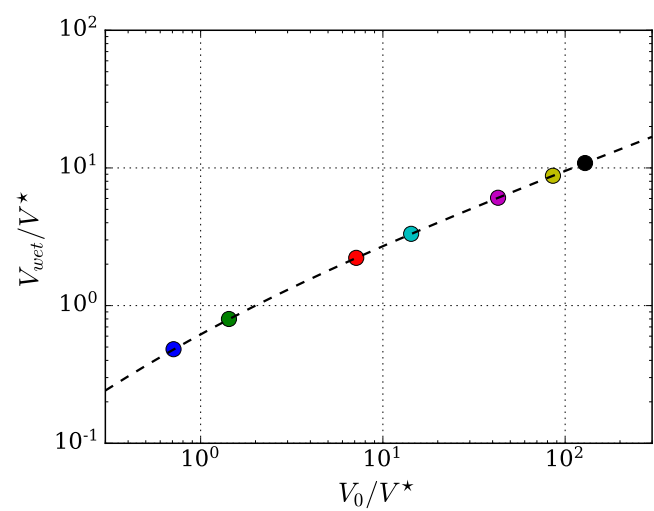

(b)

Figure 7. (a) Evolution of $L_{\text {wet }}$ as a function of $T$ given by the numerical resolution of equation (3.7) for different values of $V_{0} / V^{\star}$. The slope gives the value of $V_{\text {wet }} / V^{\star}$. (b) Fvolution of the constant impregnation velocity $V_{\text {wet }} / V^{\star}$ as function of the dimensionless plun ing speed $V_{0} / V^{\star}$. The dashed line correspond to the solution (3.10).

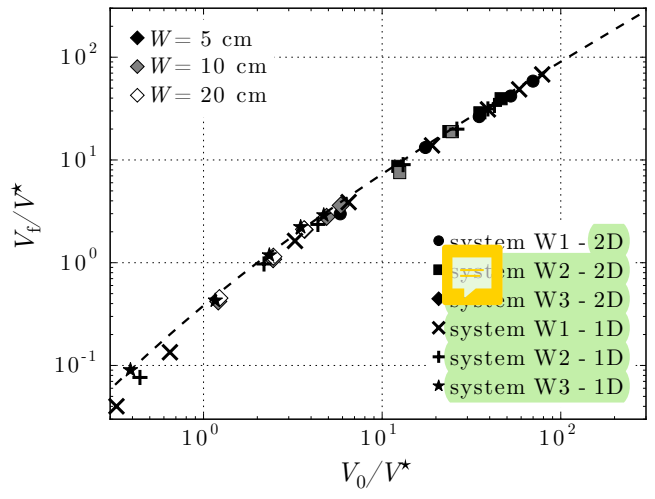

Figure 8 . Front velocity $V_{f} / V^{\star}$ as a function of the plunging speed $V_{0} / V^{\star}$ for different systems and cells widths.

where only the positive root has a physical meaning, leading to

$$
L_{w e t}=\frac{T}{2}\left[\sqrt{1+4\left(\frac{V_{0}}{V^{\star}}\right)}-1\right] .
$$

We also derived this expression in Appendix B by considering the long-time behavior of the implicit analytical solution. The solution (3.10) is plotted in dashed line in figure $7(\mathrm{~b})$ and captures the long-time behavior of the implicit solutions of equation (3.7) confirming that the long time dynamics is dominated by the pressure-driven flow and the capillary effects can be neglected.

The velocity $V_{w e t}$ corresponds to the impregnation front velocity with respect to the bottom of the porous medium and is thus related to the front velocity $V_{f}$, with respect to the free surface through

$$
V_{f}=V_{0}-V_{w e t} .
$$

The dimensionless front velocity $V_{f} / V^{\star}$ is reported as a function of the rescaled plunging velocity $V_{0} / V^{\star}$ in figure 8 , where we also show the experimental data obtained 


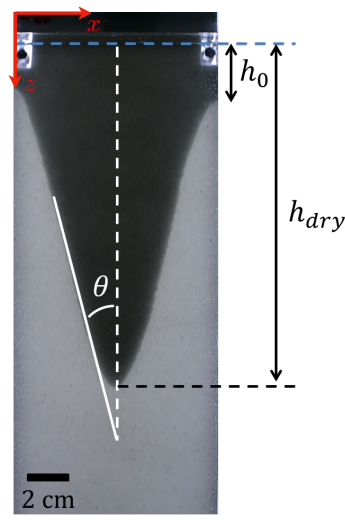

(a)

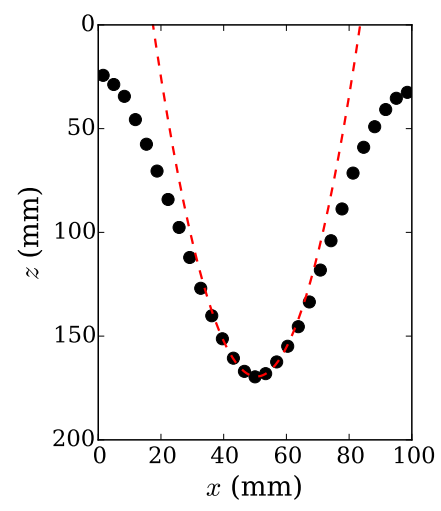

(b)

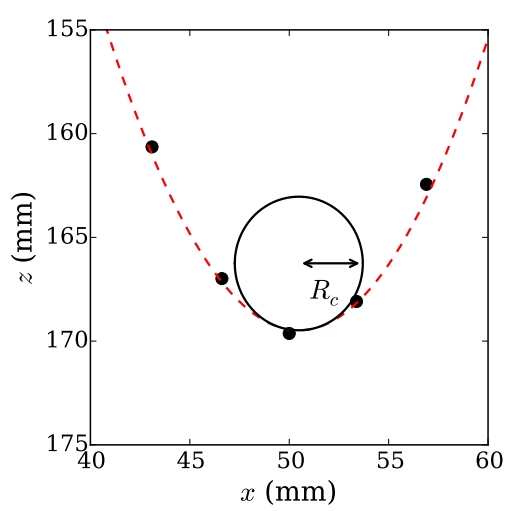

(c)

Figure 9. (a) Picture of a stationary front of impregnation obtained with the system W2, a cell of width $W=10 \mathrm{~cm}$, and a plunging speed $V_{0}=60 \mathrm{~mm} / \mathrm{s}$. (b) Impregnation profile $z(x)$ extracted from the experiment shown in (a). The red dashed line corresponds to the parabola fitting the center of the profile. (c) Close-up view of the bottom of the impregnation profile. The radius of curvature $R_{c}$ is evaluated by fitting the profile with a parabola.

for different systems of grains and widths of the cell. Additional data obtained from one-dimensional impregnation experiments are also plotted in figure 8 to compare them to the two-dimensional results in the transient regime. Both one and two-dimensional experimental results collapse on a master curve and are well captured by the analytical model developed above. These results confirm that the transient regime is governed by the impregnation from the bottom of the porous medium.

The next process to characterize is the duration of the transient regime, which is related to how the liquid impregnates the porous medium laterally.

\section{Experimental characterization of the stationary front}

We now characterize experimentally the stationary regime reached after the transient impregnation phase. In this regime, the impregnation front is stable and stationary in the frame of reference of the laboratory, whereas the porous medium is translating in the water bath. In this regime, the shape of the impregnation front results from the balance between the air entrainment by the translated porous material and the liquid impregnation coming from the side of the cell.

\subsection{Morphology of the impregnation front}

The morphology of the stationary front, which separates the wet and dry grains, is characterized by a $\mathrm{V}$-shape profile, as shown in figure 9(a). The impregnation profile exhibits a shouldering of height $h_{0}$ at the vicinity of the surface of the bath and a local curvature at the tip of the profile. The maximum length of air entrained under the liquid surface in the bath is denoted $h_{d r y}$, as represented in figure $9(\mathrm{a})$. To characterize the impregnation front, we note $\theta$ the opening angle of the profile such that $1 / \tan \theta$ is the slope. We also note $R_{c}$ the radius of curvature of the front near the tip of the profile [see figure 9(c)]. The measurements of the opening angle $\theta$ and the radius of curvature $R_{c}$ are described in figure $9(\mathrm{~b})-(\mathrm{c})$.

The evolution of $\tan \theta$, measured on each stationary $\mathrm{V}$-shape profile, is reported in figure 10 (a) as a function of the dimensionless plunging speed $V_{0} / V^{\star}$ for different systems 


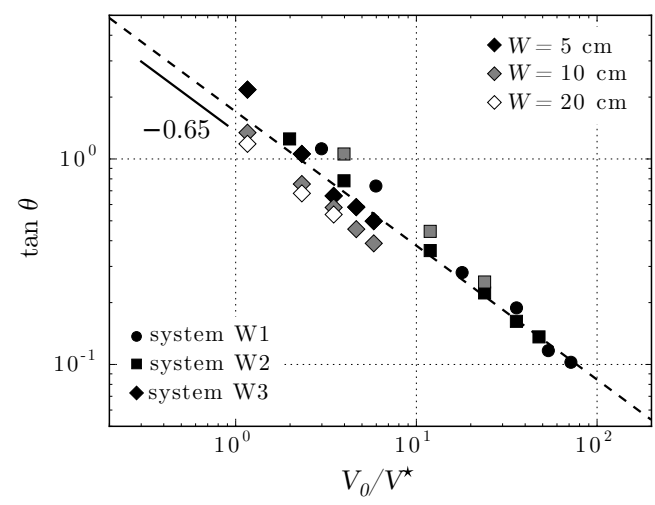

(a)

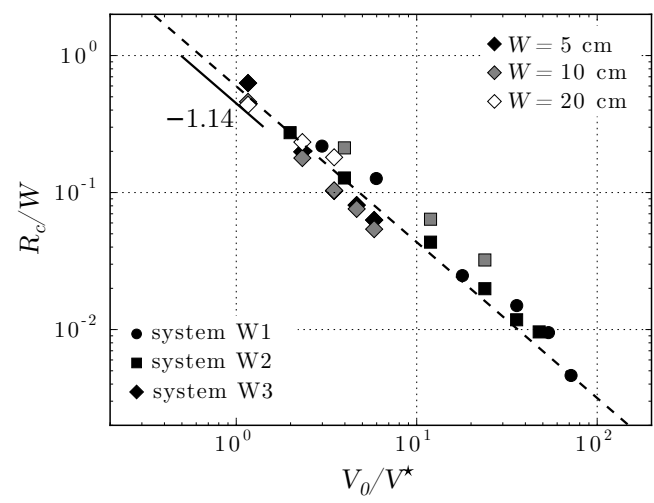

(b)

FiguRE 10. Evolution of (a) the slope $\tan \theta$ and (b) the dimensionless radius of curvature $R_{c} / W$ of the stationary profile of impregnation as a function of the dimensionless plunging speed $V_{0} / V^{\star}$ for several systems of wetting porous materials with different widths $W$. The dotted lines in both figures are the best fitting power-law.

of beads and cell widths $W$. The experimental data collapse well on a master curve confirming that the $\mathrm{V}$-shape of the impregnation profile results from the competition between the fluid penetration into the porous medium and the translation in the water bath. The experimental data are well-fitted by a power law of the dimensionless velocity:

$$
\tan \theta \propto\left(\frac{V_{0}}{V^{\star}}\right)^{\alpha} \quad \text { where } \quad \alpha=-0.65 .
$$

Similarly, the radius of curvature $R_{c}$ at the tip of the profile as a function of $V_{0} / V^{\star}$ is reported in figure 10(b). A good collapse of the data is also observed by rescaling the radius of curvature by the width of the porous medium $W$. This result indicates that the curvature is invariant by a change of scale and only depends on the aspect ratio of the impregnation front. Moreover, the experimental results suggest that the radius of curvature at the tip evolves as the inverse of the dimensionless plunging velocity:

$$
\frac{R_{c}}{W} \propto\left(\frac{V_{0}}{V^{\star}}\right)^{\gamma} \quad \text { where } \quad \gamma=-1.14
$$

Therefore, the largest the speed of immersion, the smallest the curved area is.

\subsection{Effect of wettability}

We investigate the influence of the wettability of the grains composing the synthetic porous medium on the shape of the impregnation profile. The coated grains used for these experiments (systems $\mathrm{N} 1$ and N2 in table 1 ) have a contact angle of $\theta_{c} \simeq 75^{\circ}$. The grains can thus be considered as non-wetting grains for which the capillary pressure is neglected. Moreover, a contact angle larger than about $55^{\circ}$ prevents the spontaneous imbibition in a granular packing (Raux et al. 2013). This phenomenon results from geometrical inaccessibility of the material porosity for the liquid menisci. The non-wetting porous medium is plunged in the water bath in the same conditions as the previous experiments. After a similar transient regime, a stationary profile is also observed. Contrary to the situation with wetting grains (systems W1, W2 and W3), the profile is now shifted by a much larger length $h_{0}$ and does not present any shouldering at the vicinity of the side walls as shown in the inset in figure 11(b). 
The evolution of $\tan \theta$ as a function of $V_{0} / V^{\star}$ for the non-wetting porous media is reported in figure 11(a). A transition is observed, characterized by a change of the exponent describing the evolution of $\tan \theta$ with $V_{0} / V^{\star}$ :

$$
\tan \theta \propto\left(\frac{V_{0}}{V^{\star}}\right)^{\alpha}, \quad \text { with }\left\{\begin{array}{l}
\alpha=-0.64 \text { for } V_{0} / V^{\star} \lesssim 5 . \\
\alpha=-0.5 \text { for } V_{0} / V^{\star} \gtrsim 5 .
\end{array}\right.
$$

For large plunging speeds $\left(V_{0} / V^{\star} \gtrsim 5\right)$, the exponent $\alpha$ matches well with the prediction of Nasto et al. (2016) who neglected the capillary effects and assumed a horizontal impregnation in the porous material. For small plunging speeds $\left(V_{0} / V^{\star} \lesssim 5\right)$, we find an exponent $\alpha \simeq-0.64$ similar to one reported for wetting systems in figure 10(a), which suggest that this exponent does not depend on the capillary effect. Instead, for these small plunging speeds, the impregnation front is likely shaped by the direction of the flow inside the porous medium.

The impregnation front is also characterized by an entrance depth above which the liquid does not penetrate the porous medium. This macroscopic length, $h_{0}$ corresponds to the entrance depth of the impregnation front and is also measured for different grains sizes and several plunging speeds $V_{0}$. The length $h_{0}$ increases with $V_{0}$ and typically varies from 10 to $200 \mathrm{~mm}$ in the range of velocities studied in this work with the systems N1 and $\mathrm{N} 2\left(V_{0}=1-150 \mathrm{~mm} . \mathrm{s}^{-1}\right)$.

In static situations, the entrance depth corresponds to the hydrostatic forcing required to impregnate the porous material and the metal wire mesh. The wire mesh maintains together the grains, which have a contact angle larger than the critical angle of impregnation $\theta^{\star} \simeq 55^{\circ}$ (Raux et al. 2013). Using geometrical argument, several authors have linked this depth to the accessibility of the pores by the liquid menisci (Bán et al. 1987; Lago \& Araujo 2001; Shirtcliffe et al. 2006; Raux et al. 2013). We note $h_{0}^{\star}$ the entrance depth in the static case, i.e. for $V_{0}=0$. At constant contact angle $\theta_{c}, h_{0}^{\star}$ depends only on the size of the pores (different for each system) and the nature of the wire mesh. In all the experiments presented here, the opening of the wire mesh remains unchanged. The evolution of $h_{0}-h_{0}^{\star}$ with the plunging speed $V_{0}$ is presented in figure 11(b) for the systems N1 and N2. The entrance depth $h_{0}-h_{0}^{*}$ increases as $V_{0}^{0.5}$ in a range of lengths too large to be consistent with a viscous entrainment of air along the surface of the porous material (Lorenceau et al. 2003). A possible explanation is to consider the influence of the wire mesh and the first layer of grains just behind as an additional porous material presenting a lower permeability. Due to the weak difference of size between the grid opening $\left(d_{\text {grid }}=250 \mu \mathrm{m}\right)$ and the grains diameters $\left(d_{g}=140-320 \mu \mathrm{m}\right.$ and $\left.d_{g}=280-420 \mu \mathrm{m}\right)$, the effective pore size in this layer is very small. This layer of small permeability delays the penetration of the liquid in the porous medium and leads to an increase of the length $h_{0}$. The influence of the opening size of the mesh and the physical mechanism responsible for this evolution will be discussed in section 7.2.

\section{Theoretical modeling of the impregnation profile}

In this section, we propose to model theoretically the shape of the impregnation front in the stationary regime. As a first approximation, following the approach of Nasto et al. (2016), the fluid flow in the translated porous medium is assimilated to be purely horizontal. As we shall see later, this assumption is not valid for the entire front profile but describes a fraction of the shape of the impregnation front correctly.

As previously mentioned, the impregnation front results from the competition between the capillary imbibition, the pressure-driven flows generated by the hydrostatic pressure, 


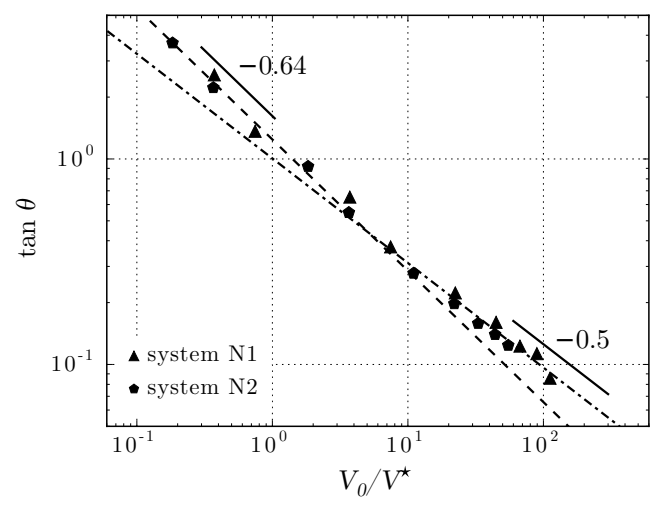

(a)

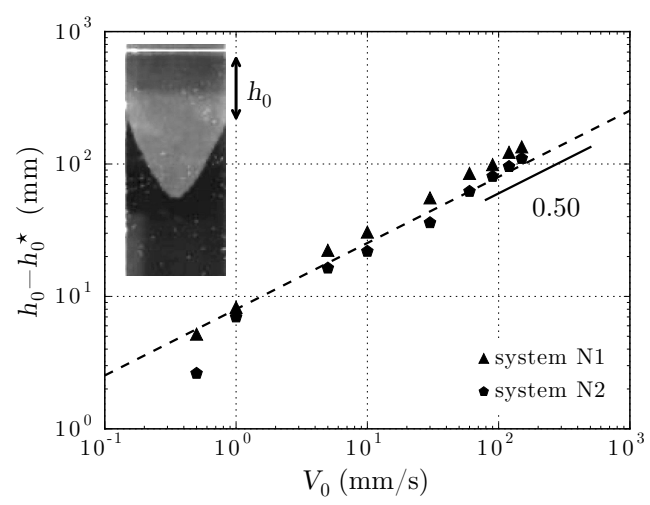

(b)

FIGURE 11. Evolution of the slope $\tan \theta$ of the stationary profile of impregnation as a function of the dimensionless plunging speed $V_{0} / V^{\star}$ for two systems of non-wetting porous media (systems $\mathrm{N} 1$ and $\mathrm{N} 2$ of contact angle $\theta \simeq 75^{\circ}$ ) and a cell of width $W=5 \mathrm{~cm}$. (b) Evolution of the lateral entrance depth $h_{0}-h_{0}^{\star}$ as a function of the plunging speed $V_{0}$ for the systems N1 and N2. The dotted lines show the best fitting power-laws.

and the translation of the porous material in the water. Because of capillary effects, the fluid velocity can be locally significant. Therefore, the assumption of laminar flows required to use Darcy's equation is not valid everywhere in the porous medium. For this reason, in this section, we determine the impregnation front profile by using Forchheimer's equation, which constitutes an extension of the Darcy's equation for larger Reynolds numbers.

\subsection{Forchheimer's equation}

For large Reynolds numbers (typically $R e_{p}>10$ ), the flow velocity in the porous material is not simply proportional to the pressure gradient. An additional correction is required to account for the inertial effects that appear in the inertial regime and add a dissipation at the pore scale. These effects are modeled by the Forchheimer's equation, written as:

$$
\nabla p=-\frac{\eta}{k} \boldsymbol{u}_{\text {Darcy }}\left(1+\frac{k \rho \beta}{\eta}\left|\boldsymbol{u}_{\text {Darcy }}\right|\right),
$$

where $\beta$ is the Forchheimer's coefficient (in $\mathrm{m}^{-1}$ ) and is typically of the order of $1 / \sqrt{k}$. For small Reynolds numbers, $k \rho \beta\left|\boldsymbol{u}_{\text {Darcy }}\right| / \eta \ll 1$ and the Forchheimer's equation (5.1) reduces to the Darcy's equation (3.4).

The Forcheimer's coefficient $\beta$ is measured for the different model porous medium using the methods presented in section 2 for the permeability measurement but now by imposing a larger pressure gradient $\nabla p$. The details of the measurements are given in Appendix A, and the values for wetting beads (systems W1, W2, and W3) are reported in table 1.

\subsection{Profile of the impregnation front}

We model the shape of the impregnation front as the result of the lateral impregnation from the side walls of the porous material. The Forchheimer's equation (5.1) reads

$$
(1-\phi) \boldsymbol{u}_{p}\left[1+(1-\phi) \frac{k \rho \beta}{\eta}\left|\boldsymbol{u}_{p}\right|\right]=-\frac{k}{\eta}(\nabla p-\rho \boldsymbol{g}),
$$


where $\boldsymbol{u}_{p}$ is the mean velocity of the liquid in the porous medium such that $\boldsymbol{u}_{\text {Darcy }}=$ $(1-\phi) \boldsymbol{u}_{p}$. We first assume that the streamlines are horizontal in the frame of reference of the porous material. We further assume that the velocity field is uniform along the horizontal direction so that $\boldsymbol{u}_{p}(x, z)=u_{x}(z) \boldsymbol{e}_{x}$, where the $z$-axis is oriented downward and the $x$-axis is perpendicular to it. The relevance of this assumption depends on the plunging speed velocity $V_{0}$ and the properties of the porous material. We shall discuss the limits of this assumption in the following sections.

Along the $x$-direction, the pressure gradient in the porous medium depends on the hydrostatic pressure, the capillary pressure and the position of the impregnation front $x_{f}(z)$. An additional length $\ell_{g}$ is added to $x_{f}$ to model the hydraulic resistance brought by the metal mesh wire, which holds the grains within the cell. The pressure gradient is thus given by

$$
\nabla p \cdot \boldsymbol{e}_{x}=-\frac{p_{c}+\rho g z}{x_{f}(z)+\ell_{g}} .
$$

The length $\ell_{g}$ is taken equal to $2 \mathrm{~mm}$ in all the calculations, leading to a good agreement between the experimental profiles and the theoretical predictions, as we shall see later. Equation (5.2) written along the $x$-direction in the frame of reference of the porous medium yields

$$
u_{x}(z)\left[1+(1-\phi) \frac{k \rho \beta}{\eta} u_{x}(z)\right]=\frac{k}{\eta(1-\phi)}\left[\frac{p_{c}+\rho g z}{x_{f}(z)+\ell_{g}}\right],
$$

We introduce $V^{\star}$ the characteristic microscopic velocity of impregnation under gravity, $\tilde{V}^{\star}$ the characteristic Forchheimer's velocity and $h_{\infty}$ the Jurin's height:

$$
V^{\star}=\frac{k \rho g}{(1-\phi) \eta}, \quad \tilde{V}=\frac{1}{(1-\phi)} \sqrt{\frac{g}{\beta}}, \quad h_{\infty}=\frac{p_{c}}{\rho g} .
$$

The equation (5.4) for the front profile thus becomes:

$$
\frac{u_{x}(z)}{V_{0}}\left(1+\frac{V^{\star}}{\tilde{V}^{2}} u_{x}(z)\right)=\frac{V^{\star}}{V_{0}}\left(\frac{h_{J}+z}{x_{f}(z)+\ell_{g}}\right) .
$$

Experimentally, $u_{x} V^{\star} / \tilde{V}^{2}$ varies from 0.1 to 10 , which further justifies using the Forchheimer's correction to accurately predict the front shape. Then, equation (5.6) leads to:

$$
\frac{u_{x}(z)}{V_{0}}=\frac{-1+\left[1+4\left(\frac{V^{\star}}{\tilde{V}}\right)^{2}\left(\frac{h_{J}+z}{x_{f}(z)+\ell_{g}}\right)\right]^{1 / 2}}{2 V^{\star} V_{0} / \tilde{V}^{2}} .
$$

\subsection{Validation of the model: Lateral impregnation}

We first compare the theoretical prediction given by the equation (5.7) to one dimensional experiments of impregnation in a horizontal porous medium translated vertically into a liquid bath [Figure 12(a)]. This configuration satisfies the assumptions used in the 1D model described previously. The grains are packed into an L-shaped glass tube of diameter $1 \mathrm{~cm}$ closed by a metal wire mesh. The porous medium is plunged at a constant speed $V_{0}$ into a water bath, as shown in figure $12(\mathrm{a})$. The evolution of the impregnation front is recorded for several plunging speeds $V_{0}$ and different systems of wetting glass beads (systems W1, W2, and W3). A time series of the impregnation fronts for the system $\mathrm{W} 3$ is reported in figure 12(b). The time series shows an initial fast impregnation at low immersion depth followed by a displacement of the impregnation front in the porous medium at a constant velocity for larger immersion depth. Furthermore, as observed 


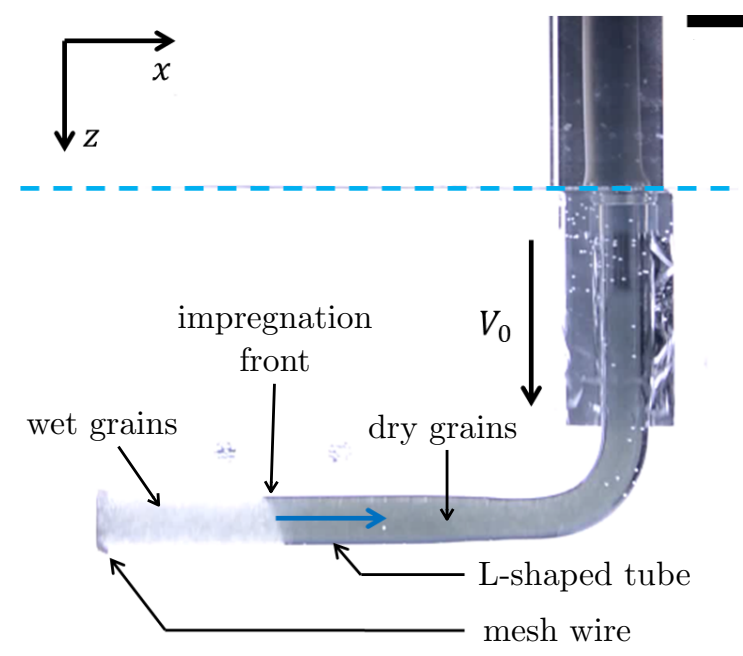

(a)

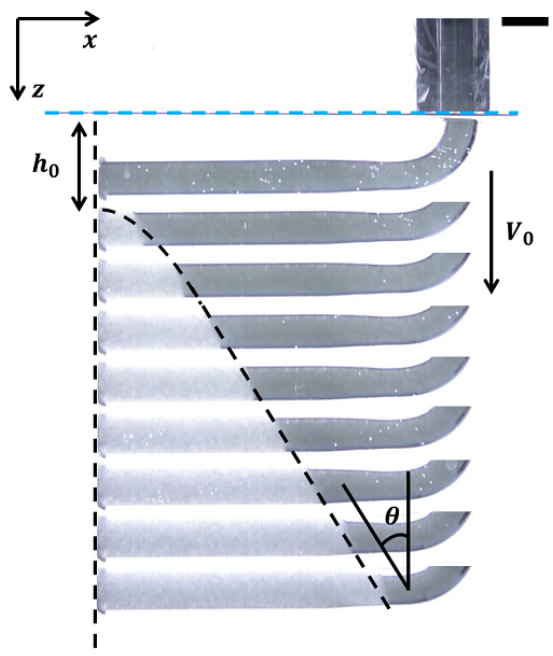

(b)

Figure 12. (a) Picture of the experimental setup used to characterized the horizontal 1D impregnation in a porous medium. The grains are packed into a L-shaped tube closed by a metal mesh wire of opening $250 \mu \mathrm{m}$. The experimental system is vertically plunged in the water bath at a constant speed $V_{0}$ and the impregnation front is visualized by the contrast of light transmission between the wet part (light) and the dry part (darker). (b) Time-series of the front propagation in the granular packing (system W3) translated at a velocity $V_{0}=60 \mathrm{~mm} \cdot \mathrm{s}^{-1}$. Scale bars are $1 \mathrm{~cm}$.

previously for non-wetting grains, the impregnation only begins for a depth larger than $h_{0}$, which weakly increases with $V_{0}$.

The porous medium is translated at a constant velocity $V_{0}$ so that its vertical position is given by $z(t)=V_{0} t$. Using $u_{z}(x)=\mathrm{d} x_{f} / \mathrm{d} t$, equation (5.7) yields to

$$
\frac{1}{V_{0}} \frac{\mathrm{d} x_{f}}{\mathrm{~d} t}=\frac{-1+\left[1+4\left(\frac{V^{\star}}{\tilde{V}}\right)^{2}\left(\frac{h_{J}+V_{0} t}{x_{f}+\ell_{g}}\right)\right]^{1 / 2}}{2\left(\frac{V^{\star} V_{0}}{\tilde{V}^{2}}\right)} .
$$

A discretization by a fourth-order Runge-Kutta method is used to solve equation (5.8) with the initial condition $x_{f}\left(t_{0}\right)=0$, where $t_{0}=h_{0} / V_{0}$. The initial condition ensures that the front profile is shifted by $h_{0}$ in the reference frame of the laboratory so that the impregnation starts at the depth $h_{0}$, as observed experimentally. This shift results from the presence of the metal wire mesh that maintains the grains in the tube and delays the liquid penetration into the porous medium. The theoretical predictions are compared to the experimental measurements in figure 13(a). A good agreement is observed with only one adjustable parameter, the length $\ell_{g}=2 \mathrm{~mm}$ modeling the hydraulic resistance induced by the metal wire mesh kept fixed in the modeling. The shouldering observed in the vicinity of the free surface is induced by the capillary pressure gradient. This effect is dominant at low depth, i.e. $h \ll h_{J}$. The linear part of the profile, observed at larger depth, i.e. $h \gg h_{J}$, results from the hydrostatic pressure gradient, which remains constant. Indeed, both the hydrostatic pressure and the distance between the impregnation front and the lateral wall grows linearly with the depth. The good agreement validates the relevance of the Forchheimer's formalism to describe the capillary flows in the porous media for the parameters considered in this study. 


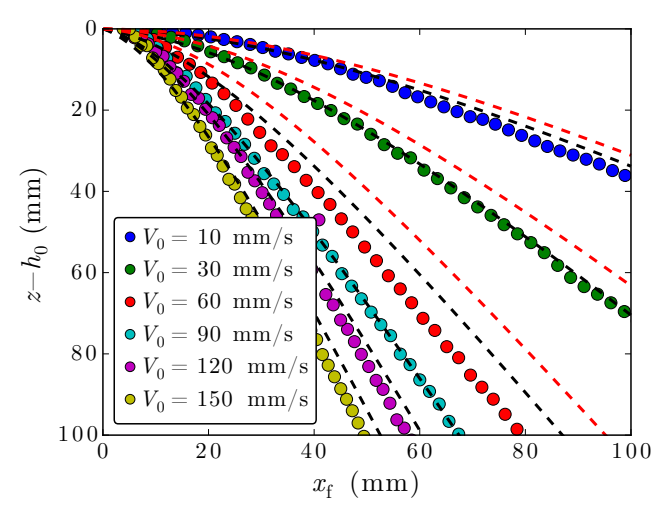

(a)

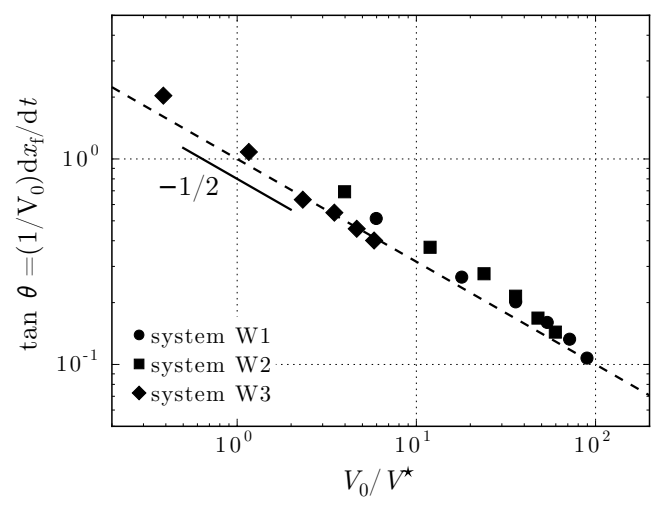

(b)

Figure 13. (a) Impregnation profiles for the experiments in the 1D geometry shown in figure 12 and the system W3. The profiles are plotted by superimposing the successive positions of the front at each time as illustrated in figure 12(b). The theoretical prediction given by equation (5.6) is plotted in black dashed line whereas the prediction of Eq. (5.10) is plotted in red dashed line. (b) Evolution of the dimensionless horizontal speed of impregnation in the linear regime as a function of the dimensionless plunging speed $V_{0} / V^{\star}$. The dashed line is the best fitting power-law.

To evaluate the importance of the inertial term in the present situation, we compare the prediction provided by the Forchheimer's and Darcy's formalisms. Neglecting the Forchheimer's correction in equation (5.6) leads to

$$
\frac{\mathrm{d} x_{f}}{\mathrm{~d} t}=V^{\star}\left(\frac{h_{J}+V_{0} t}{x_{f}+\ell_{g}}\right) .
$$

This equation, with the same initial condition $x_{f}\left(t_{0}=h_{0} / V_{0}\right)=0$, has an analytical solution:

$$
x_{f}(z)=\left[\left(\frac{V^{\star}}{V_{0}}\right)\left(z-h_{0}\right)^{2}\left(1+\frac{2\left(h_{0}+h_{J}\right)}{z-h_{0}}\right)+\ell_{g}{ }^{2}\right]^{1 / 2}-\ell_{g}
$$

where $z=V_{0} t$. This solution is plotted in red dashed lines in figure 13(a). This prediction systematically overestimates the position of impregnation front because the additional dissipation appearing when the fluid velocity is too large if not taken into account. This discrepancy is maximum near the free surface, where the capillary pressure gradient is the largest and the Frocheimer's corrective term becomes significant. For larger depths, the fluid velocity is smaller and the inertial correction becomes negligible. As a result, the impregnation profiles predicted by the solution of equation (5.6) and by equation (5.10) are parallel for $z \gg h_{J}$.

The slopes of the profiles are evaluated in the linear part for different plunging velocity $V_{0}$. The evolution of $\tan \theta$ with $V_{0} / V^{\star}$ is reported in figure 13(b). The experimental results follow a power law $\left(V_{0} / V^{\star}\right)^{-1 / 2}$, consistent with the theoretical prediction at large depths, i.e. for $z-h_{0} \ll h_{0}+h_{\infty}$. In this limit, the analytical solution reduces to

$$
\begin{aligned}
& x_{f}(z)=\left(\frac{V^{\star}}{V_{0}}\right)^{1 / 2}\left(z-h_{0}\right) \\
& \tan \theta=\frac{\mathrm{d} x_{f}}{\mathrm{~d} z}=\left(\frac{V_{0}}{V^{\star}}\right)^{-1 / 2} .
\end{aligned}
$$


This power-law also corresponds to the prediction proposed by Nasto et al. (2016) for hairy surfaces plunged vertically into a liquid. In their configuration, Nasto et al. (2016) assume that because the fibers are oriented horizontally, the induced lateral flows are only horizontal, in the direction of the fibers. Equation (5.12) is plotted in figure 13(b) and captures well the evolution of the impregnation front with the plunging speed at large depth. The good collapse between the experimental data and equation (5.12) shows that the non-linear dissipation modeled by the Forchheimer's correction is localized in the vicinity of the free surface. In this region, the pressure gradient is large because of the capillary depression and locally generates a large velocity in the porous medium. The rest of the profile can be described by the Darcy's equation.

Furthermore, we should emphasize that the previous model assumes that the flow field is purely lateral in the porous medium. Considering an infinite bi-dimensional material without capillary effects, the solution of the Darcy equations leads to this particular flow field. In the presence of capillary forces and for finite porous media, the flow direction may be modified near the shouldering. Still, this effect seems to be marginal in the present $1 \mathrm{D}$ experiments. We now compare the analytical model, validated in the $1 \mathrm{D}$ situation, to the experimental results obtained with the $2 \mathrm{D}$ porous media.

\subsection{Theoretical model and experimental $2 D$ impregnation profile}

The solution of equation (5.7) is now compared to the stationary impregnation fronts extracted from the experiments in the translated two-dimensional porous medium described in section 4. Again, equation (5.7) is solved with a fourth-order Runge-Kutta methods with the initial condition $x\left(t_{0}=h_{0} / V_{0}\right)=0$. The solutions are plotted for each systems (W1, W2 and W3) in figure 14(a)-(c) for several cell widths $(W=5,10$ and $20 \mathrm{~cm}$ ). The theoretical solution captures well the shape of the front profile in the first half of the profile, near the free surface. The discrepancy observed at the tip of the profile comes from the assumption of a semi-infinite material, which does not predict the junction between the profiles. In particular, the region at the tip, close to the junction, is more challenging to describe since the pressure field and the streamlines become complex. The focusing of the pressure gradients occurs near the tip of the impregnation profile and imposes the inflection of the streamlines. This inflection results from the isotropy of the porous material, which prevents the specific orientation of the fluid. The streamlines reorientation and the bi-dimensional effects are more significant at low values of $V_{0} / V^{\star}$ and particularly when $V^{\star}$ is large, i.e. for a porous media made of large grains. This point is consistent with the limited agreement between experimental results and theoretical prediction reported for large pore sizes (system W3) and wider cells $(W=20 \mathrm{~cm})$ shown in figure 14(c). A better prediction is observed for a narrow porous media with a low permeability [figure 14(a)-(b)].

The analytical model described previously allows determining the shape of the first half of the impregnation profile when the flow remains mostly unidirectional and horizontal. Nevertheless, this model does not predict correctly the slopes reported experimentally in figure 10(a). The calculation presented in the previous section predicts an evolution of $\tan \theta$ as $\left(V_{0} / V^{\star}\right)^{-1 / 2}$ regardless of the grains' wettability. However, the experimental results for wetting grains indicate an as evolution $\tan \theta \propto\left(V_{0} / V^{\star}\right)^{-0.64}$. This discrepancy comes both from the $2 \mathrm{D}$-effects in the flow through the porous medium, and the influence of the grains wettability since a transition in the scaling law is only observed for the nonwetting particles (see figure 11(a)). The complexity of the flow field around the tip of the impregnation profile suggests using numerical simulations to determine the entire shape of the impregnation front. 


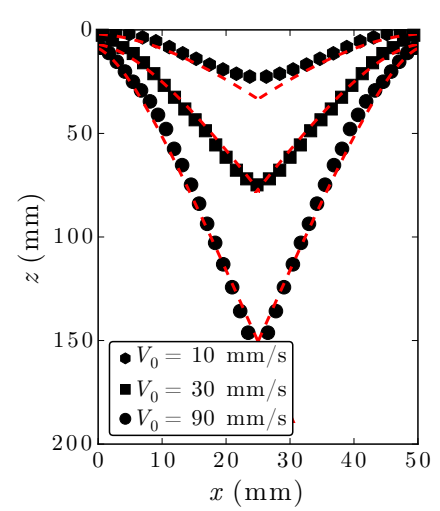

(a)

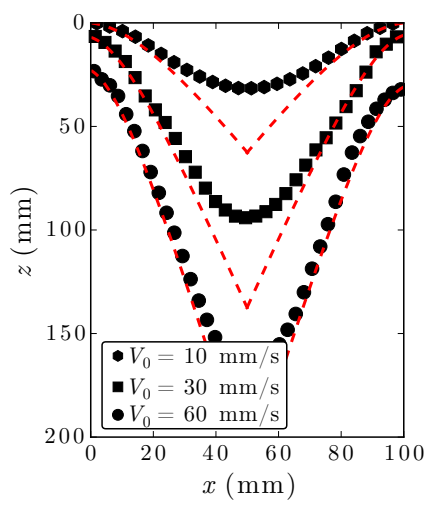

(b)

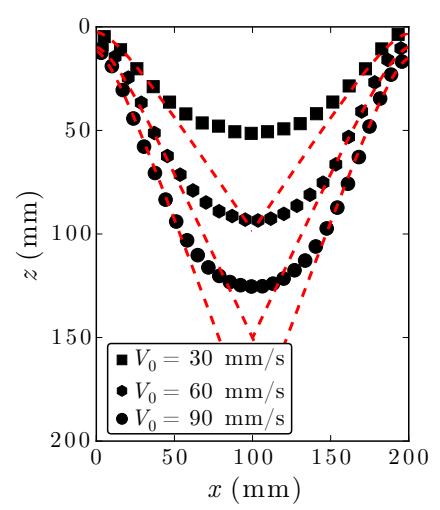

(c)

Figure 14. Profiles of the impregnation front for different experimental configurations: (a) System $\mathrm{W} 1$ and $W=5 \mathrm{~cm}$, (b) system $\mathrm{W} 2$ and $W=10 \mathrm{~cm}$, (c) system $\mathrm{W} 3$ and $W=20 \mathrm{~cm}$. The red dashed lines show the theoretical predictions given by equation (5.7).

\section{Numerical modeling of the impregnation profile in the stationary regime}

The analytical approach developed in the previous section assumed a horizontal orientation of the fluid flow to simplify the equations and obtain the shape of the profile. This assumption is only relevant in the vicinity of the free surface and is not valid near the tip of the profile, where the streamlines are deflected. In this section, we develop a numerical model to predict the shape of the entire impregnation front in the stationary regime. This approach allows us to account for the actual flow field in the porous medium.

\subsection{Details of the numerical method}

We solve numerically the pressure field $p(x, z)$ in the liquid confined in the porous medium. The liquid-air interface is imposed by the shape of the impregnation front, related to the plunging velocity. The associated pressure field is obtained from the Laplace equation and the boundary conditions. The velocity field in the porous medium, $\boldsymbol{u}_{p}(x, z)$, is then derived from the pressure field $p(x, z)$. The numerical calculation is performed in the laboratory's reference frame, where the porous medium translates into the liquid at a constant velocity, leading to a stationary impregnation front. The stationary shape of the impregnation front imposes that the flow velocity must remain constant along the front and equal to the plunging speed $V_{0}$.

The velocity field $\boldsymbol{u}_{p}=u_{x} \boldsymbol{e}_{x}+u_{z} \boldsymbol{e}_{z}$ in the porous medium is obtained from the pressure field $p(x, z)$ by solving the Darcy's equation (3.4), which can be written in dimensionless variables:

$$
\tilde{\boldsymbol{u}}=-\tilde{\nabla}(\tilde{p}+\tilde{z})
$$

where

$$
\tilde{\boldsymbol{u}}=\frac{\boldsymbol{u}_{p}}{V^{\star}}, \quad \tilde{p}=\frac{p}{\rho g W}, \quad \tilde{x}=\frac{x}{W}, \quad \tilde{z}=\frac{z}{W}, \quad \tilde{\nabla}=\frac{\partial}{\partial \tilde{x}} \boldsymbol{e}_{\boldsymbol{x}}+\frac{\partial}{\partial \tilde{z}} \boldsymbol{e}_{\boldsymbol{z}} .
$$

The Darcy's law is not relevant to describe the flow field for large Reynolds numbers. However, we have shown in the previous section that, locally, capillary effects can generate large pressure gradients, in particular near the free-surface. Here, we focus on the shape of the wetting front far from the free surface. In particular, we are interested in the complexity of the fluid flow generated in the porous medium. With this in mind, the range 
of Reynolds numbers considered along the front remains compatible with the limitations of the Darcy's law and allows us to solve the problem numerically. The combination of the dimensionless Darcy's equation (6.1) and the incompressibility of the fluid flow in the porous matrix, $\tilde{\boldsymbol{\nabla}} \cdot \tilde{\boldsymbol{u}}=0$, leads to the Laplace equation, which determines the evolution of the pressure field in the stationary regime for given boundary conditions:

$$
\tilde{\nabla}^{2} \tilde{p}=0
$$

The porous material is discretized into a finite number of rectangular elements. The Laplacian is solved on a rectangular lattice of width $N_{x}$ and height $N_{z}$ as shown in figure 15(a). The problem is symmetric along the vertical direction, we thus only solve equation (6.3) in half of the porous medium, the other part being obtained by symmetry. The discretized pressure field $p_{i, j}$ is defined at the node $i, j$ ( $i$ along the horizontal direction and $j$ along the vertical direction) and we note $\Delta$ the step of the mesh, such that $\Delta=1 / N_{x}=1 / N_{z}$. Discretizing equation (6.3) leads to

$$
\frac{1}{\Delta}\left(\frac{\tilde{p}_{i+1, j}-\tilde{p}_{i, j}}{\Delta}-\frac{\tilde{p}_{i, j}-\tilde{p}_{i-1, j}}{\Delta}\right)+\frac{1}{\Delta}\left(\frac{\tilde{p}_{i, j+1}-\tilde{p}_{i, j}}{\Delta}-\frac{\tilde{p}_{i, j}-\tilde{p}_{i, j-1}}{\Delta}\right)=0 .
$$

The stationary pressure field is obtained by solving the linear system composed of $N_{x} \times N_{z}$ equations with $N_{x} \times N_{z}$ unknowns:

$$
\tilde{p}_{i+1, j}+\tilde{p}_{i-1, j}+\tilde{p}_{i, j+1}+\tilde{p}_{i, j-1}-4 \tilde{p}_{i, j}=0 .
$$

The simulation is performed by considering a static porous medium with an imposed impregnation profile, which separates the dry region to the wet region of the porous material. The pressure at the dry-wet interface is $p_{0}-p_{c}$ where $p_{0}$ is the atmospheric pressure and $p_{c}$ the capillary pressure. The pressure along the side wall is the hydrostatic pressure $p_{0}+\rho g z$ and increases linearly with the depth. As the problem is solved on a half domain, a symmetry condition is imposed on the other side. The atmospheric pressure is taken as the reference pressure and the dimensionless boundary conditions are:

$$
\left\{\begin{array}{l}
\tilde{p}_{0, j}=j \text { at the vertical boundary } \\
\tilde{p}_{i, N_{z}}=N_{z} \text { at the bottom boundary } \\
\tilde{p}_{-i, j}=\tilde{p}_{i, j} \text { along the symmetry axis } \\
\tilde{p}_{i_{F}, j_{F}}=-\tilde{p}_{c} \text { at the liquid/air interface }
\end{array}\right.
$$

The solution of the linear system with the boundary conditions and the shape of the impregnation profile leads to the pressure field at each point of the lattice. The fluid velocity is then computed using the Darcy's law (6.1) along $\boldsymbol{e}_{x}$ and $\boldsymbol{e}_{z}$ :

$$
\left\{\begin{array}{c}
\tilde{u}_{x}=-\tilde{\nabla}_{x} \tilde{p} \\
\tilde{u}_{z}=-\tilde{\nabla}_{z} \tilde{p} .
\end{array}\right.
$$

An example of fluid velocity field $\|\tilde{u}(\tilde{x}, \tilde{z})\|=\sqrt{\tilde{u}_{x}^{2}+\tilde{u}_{z}^{2}}$ and the associated streamlines are shown in figure $15(\mathrm{~b})$ for non-wetting grains $\left(\tilde{p}_{c}=0\right)$. The vertical component of the velocity field is then evaluated along the dry-wet interface. The profile is stable and stationary if the vertical projection of the velocity $\tilde{u}_{z}$ along the interface is constant. We vary the shape of the impregnation profile until reaching a constant fluid velocity all along the impregnation front, equals to $\left\|\tilde{u}\left(\tilde{x}_{F}, \tilde{z}_{F}\right) \cdot \boldsymbol{e}_{z}\right\|=V_{0}$. The resulting profile then corresponds to the plunging velocity $V_{0}$. 


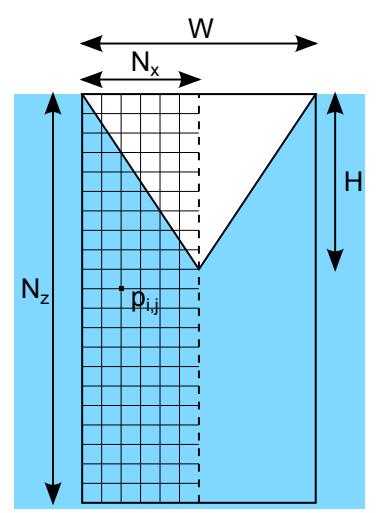

(a)

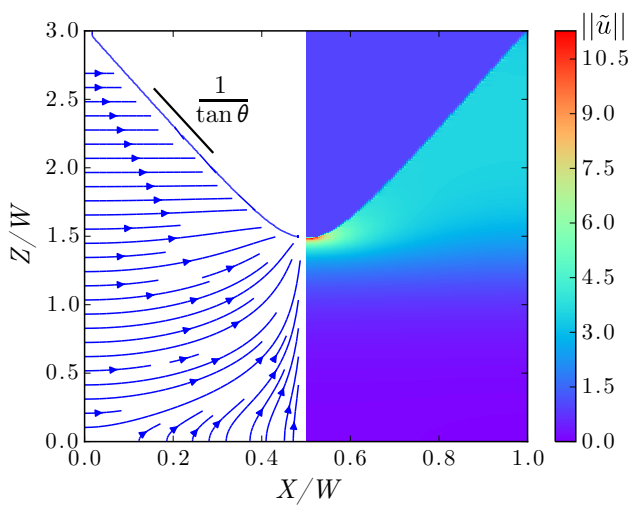

(b)

FiguRE 15. (a) Schematics of the numerical model of the stationary regime and the associated notations. (b) Examples of streamlines (left side) and velocity field (right side) observed in the stationary regime of impregnation for a non-wetting porous material $\left(p_{c}=0\right)$ and $V_{0} / V^{\star}=9.7$.

\subsection{Non-wetting grains $-\tilde{p}_{c}=0$}

We first consider the case of a porous medium composed of non-wetting grains. The capillary pressure is thus equal to zero, $\tilde{p}_{c}=0$. Therefore, the boundary condition imposed along the front profile simply becomes $\tilde{p}_{i_{F}, j_{F}}=0$. The shape of the impregnation front is modeled in the half domain $[0, \mathrm{~W} / 2]$ by the function

$$
\tilde{z}(\tilde{x})=\frac{H}{W}\left(\frac{1}{C^{n}+1}-\frac{(1-\tilde{x})^{n+1}}{C^{n}+(1-\tilde{x})^{n}}\right),
$$

which depends on three parameters $H, C$ and $n$. These parameters are chosen to have an impregnation profile along which the vertical projection of the fluid velocity remains constant to ensure the stationarity of the liquid/air interface in the reference frame of the laboratory. The parameter $H / W$ controls the aspect ratio of the profile with no curvature, the exponent $n$ adjusts the slope of the profile and the parameter $C$ modifies the curvature at the tip of the profile.

\subsubsection{Slope of the stationary profile}

The evolution of $\tan \theta$ and of the dimensionless radius of curvature at the tip of the front $R_{c} / W$, are reported in figure $16(\mathrm{a})$ and $16(\mathrm{~b})$, respectively, for different stationary impregnation profiles computed numerically. The numerical results are in good agreement with the experimental data obtained with non-wetting particles (contact angle around $\left.75^{\circ}\right)$. The values of $\tan \theta$ decreases with $V_{0} / V^{\star}$ following the scaling laws:

$$
\tan \theta \propto\left(\frac{V_{0}}{V^{\star}}\right)^{\alpha}, \text { with }\left\{\begin{array}{lll}
\alpha=-0.66 & \text { for } \quad V_{0} / V^{\star} \lesssim 5 \\
\alpha=-0.50 & \text { for } \quad V_{0} / V^{\star} \gtrsim 5
\end{array}\right.
$$

The exponents of the power laws obtained by the best fit of the numerical results are similar to the experimental values previously reported in section 4.2. The transition between the two regimes is observed at approximatively the same value of $V_{0} / V^{\star}$. Therefore, the numerical simulations correctly reproduce the evolution of the slope of the stationary profile. Besides, the radius of curvature evaluated at the tip of the impregnation profile for the numerical simulation is also in fairly good agreement with the experiments. The dimensionless radius of curvature $R_{c} / W$ decreases with the plunging 


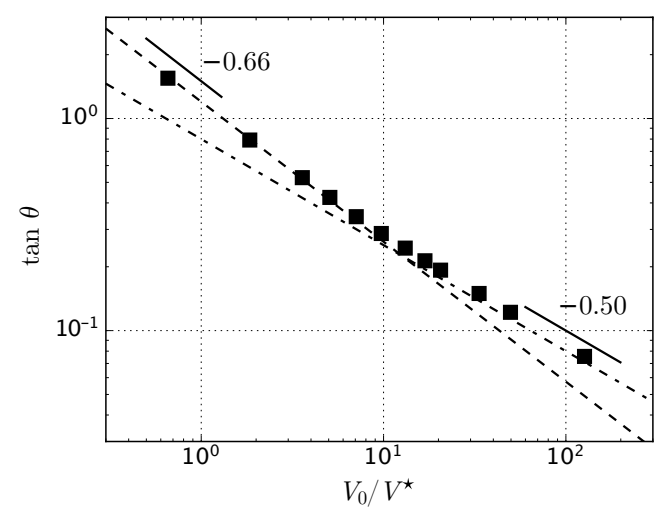

(a)

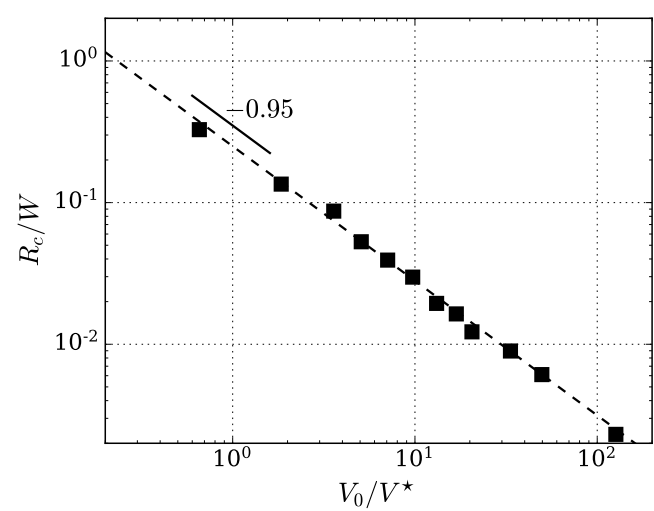

(b)

FIGURE 16. (a) Evolution of $\tan \theta$ of the stationary profile of impregnation obtained numerically as a function of the dimensionless plunging speed $V_{0} / V^{\star}$ for $p_{c}=0$. (b) Evolution of the rescaled radius of curvature $R_{c} / W$ measured at the center of the profile as a function of the dimensionless plunging speed $V_{0} / V^{\star}$. In the two figures, the numerical results are reported in black $(+)$ and the best fit is plotted in dashed lines. Numerical simulations are carried out with a mesh of width $N_{x}=100$ and length varying between $N_{z}=600$ and $N_{z}=1300$ depending on the aspect ratio $H / W$ of the impregnation front. In each case, preliminary tests are performed to ensure that the size of the domain is large enough to not influence the front velocity.

speed as

$$
\frac{R_{c}}{W} \propto\left(\frac{V_{0}}{V^{\star}}\right)^{-0.95}
$$

\subsubsection{Velocity field}

The numerical simulations allow obtaining the velocity field in the porous medium and the associated streamlines. Figures 17 (a) and 17(b) provide two examples of stationary profiles and associated flow fields for two different dimensionless plunging speeds $V_{0} / V^{\star}$. Figure 17 (a) corresponds to an immersion velocity $V_{0} / V^{\star}=0.65$, below the change of slope in the evolution of $\tan \theta$, whereas figure $17(\mathrm{~b})$, obtained for $V_{0} / V^{\star}=49.6$, reports the case beyond the transition. For $V_{0} / V^{\star}$ larger than 5 , the streamlines are mostly horizontal. The region affected by the tip effect is small and located close to the tip. In this configuration, the assumption of a pure lateral flow in the porous media is appropriate. It indeed correctly predicts the evolution of the slope $\tan \theta$ of the front profile with the dimensionless plunging speed. Conversely, for $V_{0} / V^{\star}$ smaller than 5 , as illustrated in figure 17(a), streamlines are not horizontal but instead bended around the front. The flow reorientation induces a flattening of the front profile and an increase of $\tan \theta=\mathrm{d} x / \mathrm{d} z$. The radius of curvature can reasonably be interpreted as a characteristic length of the disturbance generated by the tip of the impregnation front. The larger the radius of curvature, the broader the area of streamlines deformation is.

The numerical simulations suggest that the transition observed for the slope of the impregnation profile results from a change of flow morphology inside the porous medium. The exponent $\alpha=-0.66$ observed at low plunging speed is the result of the deformation of the streamlines of the flow on a characteristic length that increases as $1 / V_{0}$. Below a rescaled critical radius of curvature $R_{c} / W \simeq 5 \times 10^{-2}$, the change of direction of the streamlines inside the porous medium remains confined near the tip of the impregnation profile. It only affects the shape of the impregnation front in this region but not the slope. The critical radius of curvature corresponds to a slope $\tan \theta$ of about 0.5 . It means 


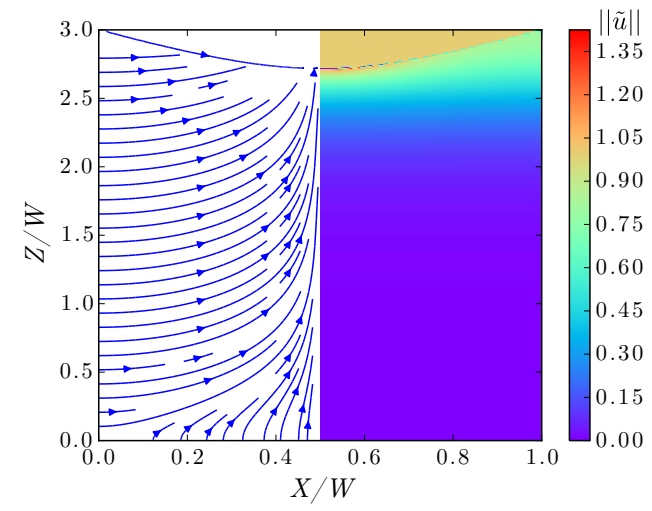

(a)

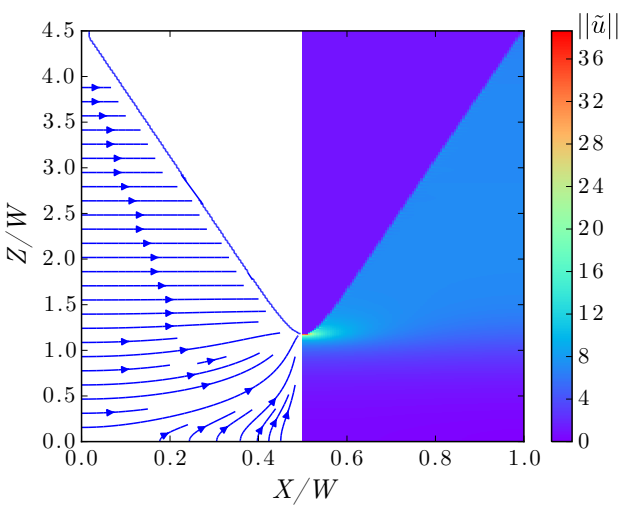

(b)

Figure 17. Streamlines (left side) and amplitude of the velocity field (right side) for two stationary impregnation fronts determined numerically for a non-wetting porous material $\left(p_{c}=0\right)$ and a dimensionless plunging velocity (a) $V_{0} / V^{\star}=0.65$ and (b) $V_{0} / V^{\star}=49.6$.

that the transition between the two regimes occurs for impregnation fronts presenting an aspect ratio smaller than 0.5 , which is consistent with the experimental observations.

\subsection{Capillarity: wetting grains $-\tilde{p}_{c}>0$}

The previous numerical simulations were performed without capillary pressure and revealed an evolution of the flow morphology with the plunging velocity $V_{0}$. This observation is consistent with the experiments carried out with non wetting grains (systems N1 and N2). Nevertheless, for wetting grains (systems W1, W2 and W3), this transition is not observed in the same range of plunging speeds. To understand the difference, we now account for the capillary pressure along the impregnation front in the numerical simulation. The new boundary condition at the dry/wet interface is $\tilde{p}_{i_{F}, j_{F}}=-\tilde{p}_{c}$, with $\tilde{p_{c}}>0$ for wetting grains. We have observed experimentally that the shape of the stationary impregnation front exhibits two curvatures in opposite direction: one close to the free surface of the water bath and another near the tip of the front. To capture the numerical stationary fronts, we adjust the shape of the boundary in the porous material using now the function:

$$
\tilde{z}(\tilde{x})=\frac{H}{W}\left(\frac{1}{C_{1}{ }^{n}+1}-\frac{(1-\tilde{x})^{n+1}}{C_{1}^{n}+(1-\tilde{x})^{n}}+(1-\tilde{x}) \frac{\tilde{x}^{n+1}}{C_{2}{ }^{n}+\tilde{x}^{n}}\right) .
$$

The last term, with the parameter $C_{2}$, is added to reproduce the upper part of the profiles. Again, the parameters $H, C_{1}, C_{2}$ and $n$ are optimized to get an impregnation profile along which the vertical projection of the fluid velocity remains constant. Note that at low plunging velocities $V_{0}$, the fluid may move upward close to the top corner because of the capillary forces. We adapted the numerical code by increasing the size of the domain above the free surface of the bath with a no flux boundary condition at the material/air interface for $\tilde{z}<0$. The capillary effects are expected to be important when the capillary speed, $V_{c}^{\star}=k p_{c} /(\eta W)$, is comparable to the plunging speed $V_{0}$. The simulations are performed with $\tilde{p}_{c}=1$, corresponding to $p_{c}=\rho g W$. As a result, the capillary speed $V_{c}^{\star}$ is equal to the characteristic velocity $V^{\star}$. The upward capillary impregnation is only observed for $V_{0} \ll V^{\star}$ ( $V_{0} / V^{\star}<50$ in practice).

An example of velocity profile is reported in figure 18(a). From the stationary front, we extract the value of $\tan \theta$ at the middle depth for varying $V_{0} / V^{\star}$ as shown in figure $18(\mathrm{~b})$. 


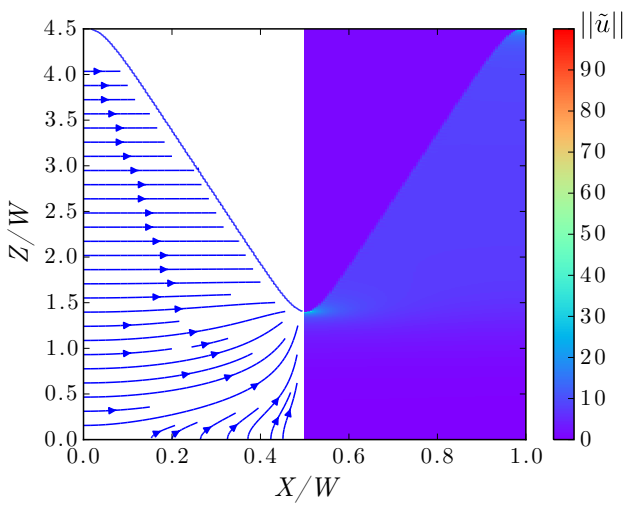

(a)

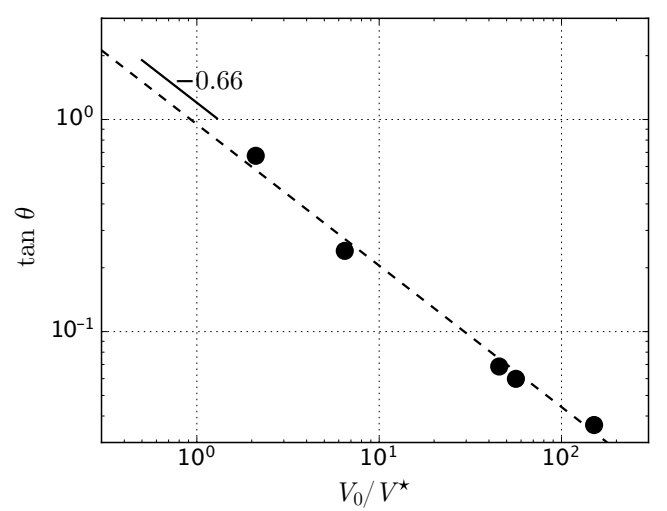

(b)

FiguRE 18. (a) Streamlines and velocity field obtained numerically for the stationary impregnation front for a wetting porous material, $\tilde{p}_{c}=1$ and $V_{0} / V^{\star}=44.4$. (b) Evolution of the slope $\tan \theta$ measured on the numerical stationary profiles as a function of the imposed dimensionless velocity $V_{0} / V^{\star}$ for $\tilde{p}_{c}=1$. The dotted line is the best fit of the numerical data $(\times)$ by a power law of exponent 0.66 .

For wetting grains, we observe that $\tan \theta \propto\left(V_{0} / V^{\star}\right)^{-0.66}$ over the whole range of $V_{0} / V^{\star}$ investigated here. This evolution is in quantitative agreement with the experimental measurements, as reported in figure 10. The conservation of the power law for high plunging speed $V_{0} / V^{\star}$ is thus related to the capillary effects, which constrained the shape of the impregnation front near the free surface.

\section{Discussion and extension to confined granular jet}

In this section, we first discuss the main results for the morphology of the impregnation front in the stationary regime. These results and predictions for porous media are then compared to the observation of a confined granular jet falling into a water bath.

\subsection{Front morphology}

The morphology of the impregnation front results from a combination of hydrostatic, geometrical, and capillary effects, each predominant in different regions of the impregnation front, depending on the plunging velocity. The numerical simulations allowed us to decompose the different contributions. The capillary effects are predominant for wetting grains at low depth, i.e. for $z<h_{\infty}=p_{c} /(\rho g)$. The capillary effects induce large pressure gradients near the liquid surface and generate a shouldering of the impregnation front profile. In this region, the low Reynolds flow assumption is not valid, and additional inertial dissipation must be accounted for to capture the amplitude of the capillary imbibition. For larger plunging depth, i.e. $z \gg h_{\infty}=p_{c} /(\rho g)$, the hydrostatic pressure is the dominant mechanism and increases linearly with the depth. The numerical simulations revealed that the flow profile induced by the hydrostatic pressure gradient is mainly horizontal. The flow profile leads to the linear portion of the impregnation front, between the capillary shouldering at the top and the front tip. In the region surrounding the front tip, the fluid flows coming from each side meet. The shape of the front tip is mainly a geometrical effect. The streamlines have to bend, which strongly affects the shape of the front. The radius of curvature $R_{c}$ of the front tip can be seen as the characteristic length of the bending of the streamlines. Indeed, the streamlines have to 
bend on a distance $R / \tan \theta$ to reach the curve tip. Moreover, the numerical results reported in figures $1 \delta(\imath)$ show that the upwards streamlines arise from the iso-pressure $p=\rho g W /(2 \tan \theta)$, which are parallel to the impregnation front. The resulting vertical pressure gradient can be written as $|\nabla p| \simeq \rho g W / R_{c}$. Using the Darcy's equation at the front tip, where the upwards speed is equal to $V_{0}$, we can write:

$$
V_{0} \simeq \frac{k}{\eta(1-\phi) \nabla p} \quad \text { so that } \quad \frac{R_{c}}{W} \simeq\left(\frac{V_{0}}{V^{\star}}\right)^{-1} .
$$

This prediction is in satisfactory agreement with the experimental measurements and the nivi nerical simulation for the evolution of the radius of curvature at the tip as a function of the dimensionless plunging speed $V_{0} / V^{\star}$.

The evolution of the main slope of the front shape is a consequence of the combination of these different effects. By considering a pure horizontal impregnation without capillary effect in a semi-infinite porous medium, Nasto et al. (2016) have also obtained a scaling law $\tan \theta \propto\left(V_{0} / V^{\star}\right)^{-1 / 2}$. This prediction is observed experimentally and numerically in our case but only for a porous medium composed of non-wetting grains at high plunging speeds. For wetting grains where capillary effects are present, a transition to an exponent $-0.65 \pm 0.01$ is observed in the experiments and the simulations at low plunging speeds. The change of exponent is induced by the tip of the front where impregnation fronts from each side meet. The characteristic size of the tip increases as the inverse of the plunging speed, as illustrated in figure 16(b). At low velocity, this geometrical contribution affects the linear portion of the front and flattens it. The resulting slope, $1 / \tan \theta$, becomes lower than the semi-infinite case, as shown in figure 16(a). Furthermore, the transition is not present for porous media composed of wetting grains, as observed hoth experimentally and numerically. This observation suggests that the exponent 0,$6 ; \pm 0.01$ at high plunging speed results from the addition of capillary effects to the hydrostatic and geometrical effects. As the front shape confined by the curve tip, the capillary shouldering increases the slope of the front compared to the non-wetting case.

\subsection{Origin of the entrance depth $h_{0}$}

We now discuss the evolution of the impregnation depth $h_{0}$, illustrated in figure 19 (a), with the plunging speed $V_{0}$, observed for non-wetting grains. This length corresponds to the dry length of porous medium under the liquid/air interface of the liquid bath. Our experiments have shown that $h_{0}$ increases as $V_{0}^{0.5}$, as reported in figure 11(b). This effect can be described by considering the role of the metal mesh wire that keeps the grains in the cell. As the mesh of the grid, of opening 250 microns, is comparable to the size of the particles, the arrangement of the grains on the mesh wire leads to effective pores of smaller size. This layer then behaves locally as an effective porous medium of lower permeability over a thickness $\delta$. The thickness $\delta$ can be assumed to be of the order of the grain size, so that we consider $\delta \sim d_{g}$ in the following. We note $k_{g}$ the local effective permeability of this layer. As this permeability is lower, the fluid flowing through it is subject to a delay. This effect generates an apparently dry height $h_{0}$ in the experiments, as illustrated schematically in figure 19(a).

The flow in this layer results from the hydrostatic pressure gradient. Since the size of the effective pores in the layer is rather small and the grains are not wetting, the flow can be modeled by the Darcy's equation. In this case, the impregnation profile is a straight line of slope

$$
\tan \theta=\frac{\delta}{h_{0}-h_{0}^{\star}}=\left(\frac{V_{0}}{V_{g}^{\star}}\right)^{-1 / 2}
$$


where $V_{g}^{\star}$ is the characteristic impregnation velocity under gravity in the layer of thickness $\delta$, and $h_{0}^{\star}$ is the forcing depth of impregnation in the static regime. This velocity is related to the effective permeability though the the Carman-Kozeny's equation (Carman 1937)

$$
V_{g}^{\star}=\frac{k_{g} \rho g}{(1-\phi) \eta}=\left(\frac{d_{\mathrm{eff}}}{d_{g}}\right)^{2} \frac{k \rho g}{(1-\phi) \eta}=\left(\frac{d_{\mathrm{eff}}}{d_{g}}\right)^{2} V^{\star} .
$$

Assuming that the compacity $\phi$ remains the same in each region, the permeability is proportional to the square of the pores size. The diameter $d_{\text {eff }}$ corresponds in this case to the diameter of the effective pores created by the distribution of the grains near the grid and depends on the ratio between the opening of the mesh wire and the grain diameter. The evolution of $h_{0}-h_{0}^{\star}$ as a function of $V_{0}$ is reported in figure 19(b) for different values of mesh wire opening. The value of $h_{0}-h_{0}^{\star}$ increases when decreasing the size of the wire mesh opening, which corresponds to a decrease of the effective local permeability. As a result, the impregnation depth, $h_{0}-h_{0}^{\star}$, can be written as a function of the plunging velocity:

$$
\frac{h_{0}-h_{0}^{\star}}{d_{g}} \simeq \frac{d_{g}}{d_{\mathrm{eff}}} \sqrt{\frac{V_{0}}{V^{\star}}} .
$$

The experimental data $d_{\text {eff }}\left(h_{0}-h_{0}^{\star}\right) / d_{g}{ }^{2}$ are plotted as a function of $V_{0} / V^{\star}$ in figure $19(\mathrm{c})$ for different grains sizes of non-wetting systems (N1 and N2) and metal mesh wire. A good collapse of the data is obtained for a value of $d_{\text {eff }}$ between 5 and 10 microns for all mesh wire considered here. The order of magnitude of the effective pore size is consistent with the space created by the arrangement of the grains on the grid.

\subsection{Comparison with the confined granular jet}

We now consider the initial situation of a dense granular jet entering a water bath, as illustrated in figure 1. We perform granular jet experiments in conditions close to those of the porous media. We consider a free jet of width $\mathrm{W}=5 \mathrm{~cm}$ confined between two PMMA plates at the rear and front, separated by a distance $12 \mathrm{~mm}$ and by two lateral wire mesh [figure 20(a)]. The jet is quasi-bidimensional, allowing us to visualize the impregnation front, similar to the situation of a confined porous medium. Figure 20(b) illustrates the morphology of the dense granular jet of glass beads of diameter $d_{g}=400$ $460 \mu \mathrm{m}$ entering the water bath. The jet slows down when crossing the water-air interface and permeates while remaining dense. A V-shape impregnation front is observed for the granular jet, similar to the impregnation of the porous media reported previously. After being wetted by the liquid, the grains form a "granular plug", composed of dry grains and immersed grains, from which the bottom grains wetted by the liquid escape at a flow rate $Q_{c}$. We should emphasize that even if the transient free-fall velocity of the grains is initially large, a dense flowing column, shown in figure 20(b), is observed as soon as the granular plug is formed. Besides, the velocity $V_{0}$ in the case of a granular jet in the steady-state is selected by the system itself and does not depend on the height at which the grains are initially released. Below the dense, dry phase, the wet grains form a dense jet instead of falling freely as a dilute suspension. This immersed jet configuration may be enhanced by recirculations of fluid on both sides of the jet of grains dispersed into water.

Following the same analysis as in the previous sections, we measure the morphology of the impregnation front in the steady-state for all the systems of wetting grains: the mean slope $\tan \theta$ and the mean radius of curvature at the tip $R_{c}$. The results are reported in figure 21(a)-(b), where we have also added the experimental results obtained previously 


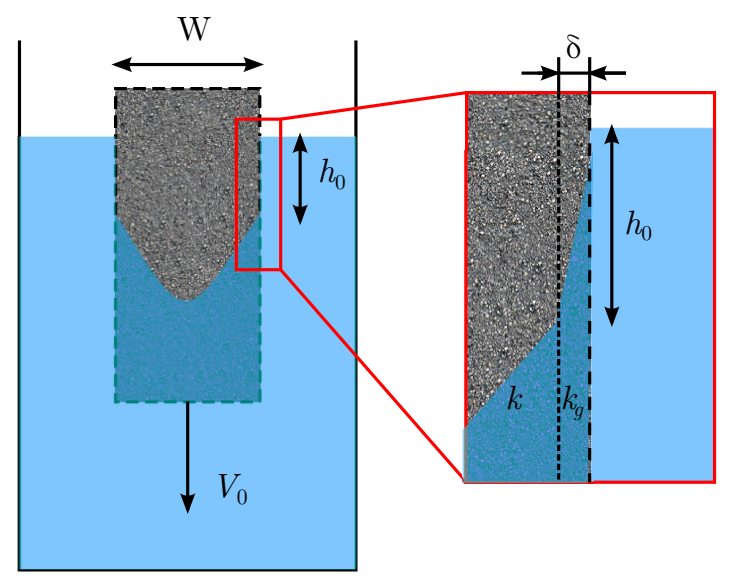

(a)

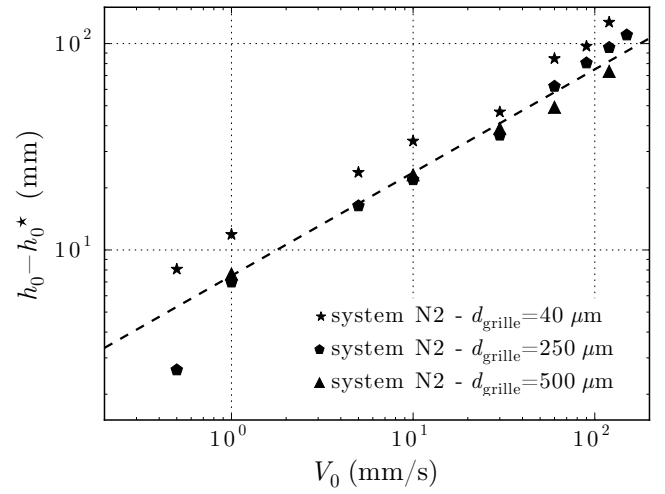

(b)

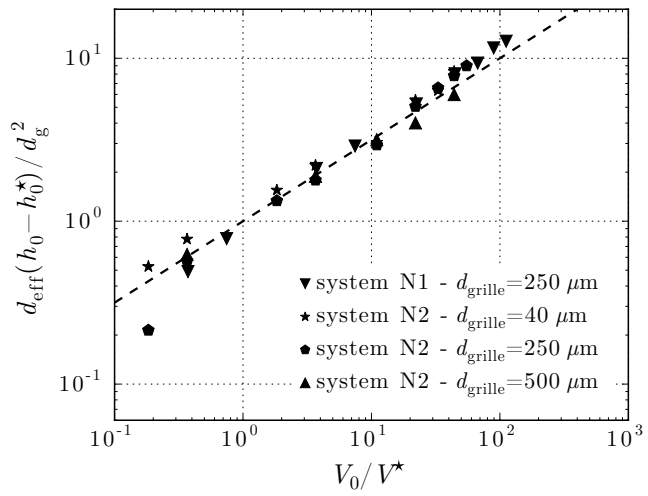

(c)

Figure 19. (a) Schematic illustration of the physical origin of the impregnation depth $h_{0}$. The length $h_{0}^{\star}$ represents the hydrostatic forcing depth required to get the non-wetting grains wet $\left(\theta>\theta^{\star}=55^{\circ}\right)$. The arrangement of grains on the metal grid is modeled by a thin layer of thickness $\delta$ with a lower permeability $k_{g}$. (b) Evolution of the impregnation depth $h_{0}-h_{0}^{\star}$ as a function of the plunging speed $V_{0}$ for grains of diameters $280-420 \mu \mathrm{m}$ (system N2) and different grid openings. (c) Evolution of the rescaled impregnation depth $d_{\text {eff }}\left(h_{0}-h_{0}^{\star}\right) / d_{g}{ }^{2}$ for different grains sizes (systems N1 and N2) and different grid openings. The dashed line shows the prediction given by equation (7.4).

for the translating porous media. The velocity $V_{0}$ of the granular jet in the steady-state is normalized by the characteristic velocity $V^{\star}=k \rho g / \eta$.

The values obtained for the slope of the granular jet, $\tan \theta$, are similar to those obtained with the porous media. This result supports our initial approach to approximate the dense jet by a porous media translating into water at the velocity $V_{0}$. The radius of curvature at the tip, $R_{c}$, seems to be more significant in the case of the confined jet than for the porous media. Qualitatively, we expect the shape of the interface between the suspension and the water to be modified the shape of the impregnation front. Indeed, the shape of the impregnated region in the jet configuration is slightly different, and the liquid needs to flow through a shorter distance in a porous medium before reaching the dry region [Figure 20(b)]. Therefore, the position of the tip of the impregnation front for the jet should be slightly higher, while the slope of the profile should remain similar 


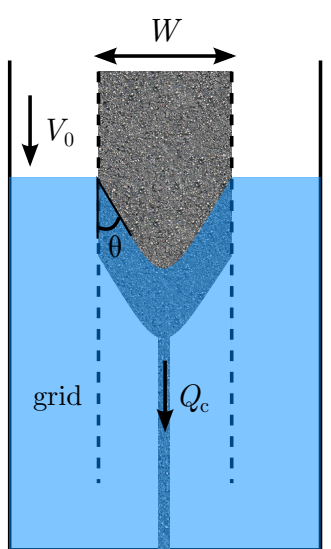

(a)

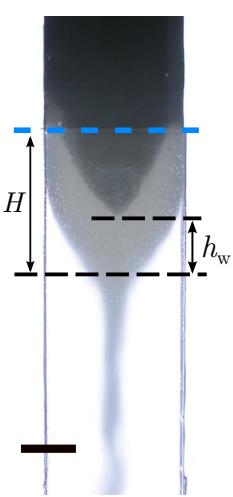

(b)

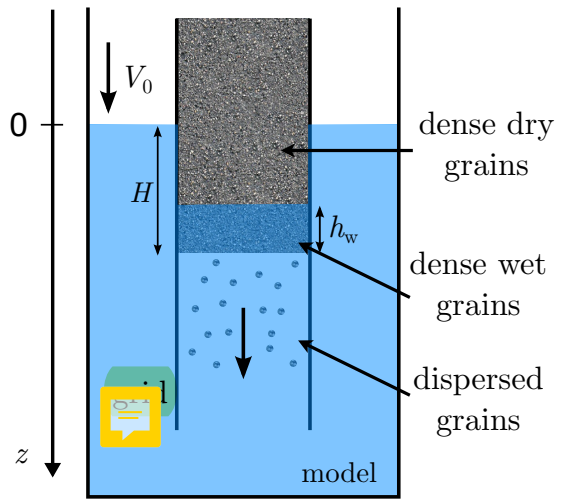

(c)

Figure 20. (a) Schematic of a granular jet entering into water, confined by a lateral wire mesh of aperture $250 \mu \mathrm{m}$. (b) Steady-state of the confined granular jet of beads of diameter 400-460 $\mu \mathrm{m}$. Scale bar is $2 \mathrm{~cm}$. (c) $1 \mathrm{D}$ model of the confined jet, assuming that the motion of the grains is only vertical and three different zones are on top of each other: dense dry grains, dense wet grains, and grains dispersed in water.

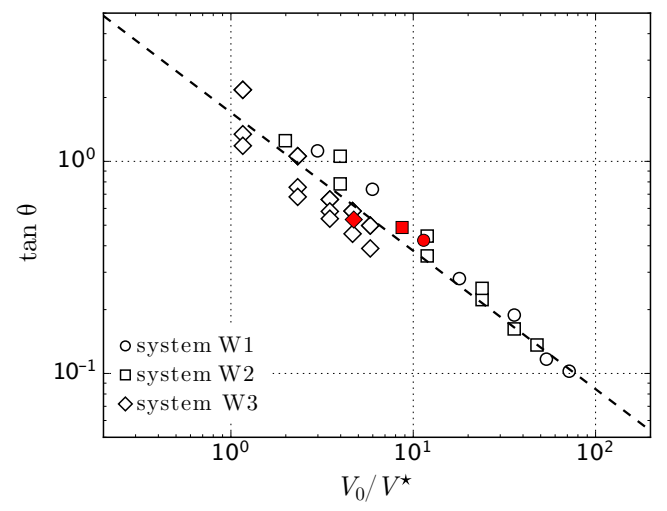

(a)

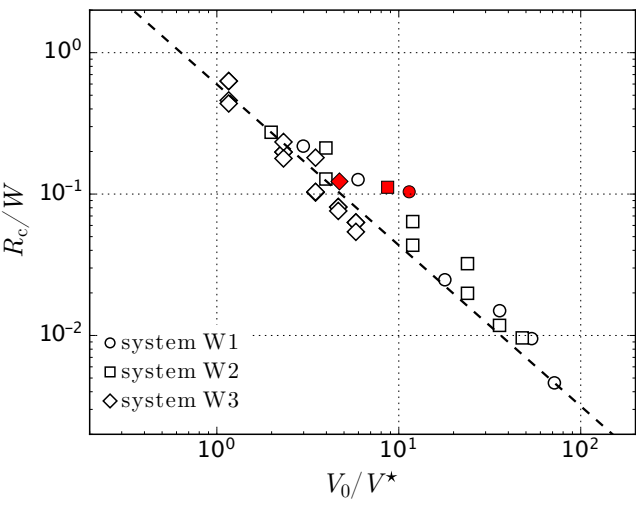

(b)

Figure 21. (a) Evolution of the mean slope $\tan \theta$ of the stationary front as a function of the dimensionless velocity $V_{0} / V^{\star}$ (red symbols). The experiments performed with the model porous media are also reported. (b) Evolution of the mean radius of curvature at the tip of the steady profile $R_{c} / W$ as function of the normalized flowing velocity $V_{0} / V^{\star}$ (red symbols). The open symbols are the same as in figure 10 .

between the two situations for the same velocity. As a result, the entry of the porous media was characterized by a V-shape, whereas in the case of the jet, the impregnation profile becomes closer to a U-shape with the same profile slope.

The remaining question is to understand the selection of the velocity $V_{0}$ at which the grains enter into water in a steady state. Figure 20(c) shows the configuration of the simplified model considered. We simplify the problem by assuming a flat interface between the wet and dry grains and between the water and the wet grains. Moreover, the jet is considered to be dense up to the water/wet grains interface, where the grains can freely escape and flow as a suspension without disturbing the water. The relative velocity between the grains and the liquid inside the pores is equal to $V_{0}$, i.e. the flowing 
velocity of the grains. Therefore, our model is based on three equations. First, the flow dynamics of the water in the pores is governed by the Darcy's equation (3.4), where the pressure gradient is given by the pressure at the bottom and the capillary pressure at the dry/wet interface. The second equation corresponds to the balance of the forces on the granular media composed of the dense column of grains and the water inside the pore. The third equation is obtained by assuming that the upward flux of liquid at the interface between the wet grains and the water is adjusted to the limit velocity allowing the immersed grains to fall. Indeed, if the fluid flux is larger, or smaller, then the length of the immersed media will grow by blocking the grains, or decrease by the quick production of a suspension, respectively.

The first equation relates the relative velocity of the water inside the pores with respect to the grains, $u_{p}$, to the water pressure at the bottom:

$$
u_{p}=-\frac{k}{\eta(1-\phi)}(\nabla p-\rho g)=-\frac{k}{\eta(1-\phi)} \frac{p_{c}+\rho g\left(H-h_{w}\right)}{h_{w}} .
$$

The second equation, which describes the force balance on the grains and the liquid in the pores, involves the weight of the grains, the air and liquid pressure at the bottom, and the friction on the vertical walls. Since the grains are confined in a gap of $e=12 \mathrm{~mm}$ between two plates of width $W=5 \mathrm{~cm}$, their apparent weight at the bottom of the column is modified by the solid friction at the walls. Janssen (1895) has described this effect in the case of homogeneous dry grains. To evaluate the granular pressure $p_{g}$ and the friction on the walls in the layer of the immersed grains, we need to account for all the forces acting on the grains: the buoyancy and the drag force of the liquid. In the steady-state, the resulting equilibrium equation for a horizontal slice of wet grains is:

$$
-\frac{p_{g}}{\lambda}+\phi \rho_{g} g-\frac{\mathrm{d} p_{g}}{\mathrm{~d} z}-\phi \frac{p_{c}+\rho g H}{h_{w}}-(1-\phi)\left[\frac{p_{c}+\rho g\left(H-h_{w}\right)}{h_{w}}\right]=0,
$$

with

$$
\lambda=\frac{e W}{2(e+W) K \mu},
$$

where $\mu$ is the friction coefficient between the grains and the walls and is typically taken equal to 0.15 (Shojaaee et al. 2012). The parameter $K$ corresponds to the redirection coefficient, which is the proportionality between the vertical and the horizontal pressures, and the value is typically around 0.8 (Vanel \& Clément 1999). The three first terms in equation (7.7) correspond to the terms derived by Janssen (1895): the solid friction force on the walls, the weight of the grains and the differential of the granular pressure. For dry grains, these terms lead to the saturation of the apparent pressure (or apparent weight) at the bottom of a long column $p_{J d r y}=\lambda \rho_{g} g$. The fourth term corresponds to the effect of the water pressure gradient on the grains and is equivalent to the static Archimede force. The fifth term corresponds to the drag force induced by the vertical Darcy flow in the grains. The granular pressure can be calculated with the appropriate boundary condition at the dry/wet interface. Due to capillary effects, the granular pressure is subjected to a discontinuity starting from the assumed saturated-granular-pressure in the dry phase $p_{g}=p_{J d r y}+p_{c}$. Applying a global force balance on the entire system in the steady-state allows us to find a second relation between $H$ and $h_{w}$.

Finally, we assume that the velocity $u_{p}$ can be estimated from experiments performed in fluidized bed (Campos \& De Carvalho 1992). For excessive flux, the grains are entrained to the top of the column and form a dense granular plug. Decreasing the fluid velocity, Campos \& De Carvalho (1992) estimated the critical velocity supporting a dense granular plug at the top of a fluidized column to be proportional to the Ergun velocity, i.e. the 


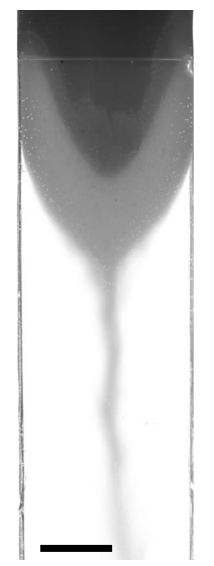

(a)

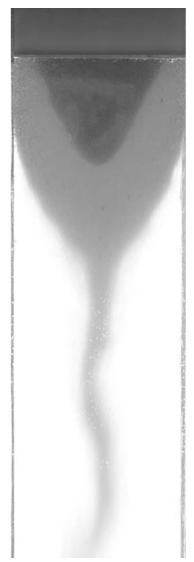

(b)

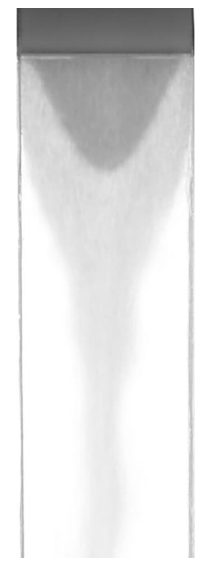

(c)

FiguRE 22. Steady morphologies of the confined jets for wetting grains of increasing diameter: (a) system W1, (b) system W2 and (c) system W3. Scale bar is $2 \mathrm{~cm}$.

minimum fluidizing velocity:

$$
(1-\phi) u_{p}=2.5 u_{\text {Ergun }}=2.5 \frac{k \phi}{\eta}\left(\rho_{g}-\rho\right) g .
$$

We can solve the system of three equations numerically. Equation (7.8) sets the dimensionless velocity to a fixed value for all grains: $V_{0} / V^{\star}=2.5 \phi\left(\rho_{g}-\rho\right) / \rho \approx 2.25$. Despite the simplified approach, the experimental velocities reported in figure 21 are of the same order of magnitude. The predicted values the height of immersed grains before this dispersion, $H$, are $4.2 \mathrm{~cm}, 4.3 \mathrm{~cm}$, and $4.7 \mathrm{~cm}$ for increasing bead diameters, and the predicted values of $h_{w}$ are $3.2 \mathrm{~cm}, 3 \mathrm{~cm}$, and $2 \mathrm{~cm}$, respectively. Those values are also in qualitative agreement with the values that can be estimated from figures 22(a)-(c), where all the systems exhibit a height of dry grains in a comparable range.

While this model is rather simplified, the results are in good agreement with the experimental values. This encouraging comparison calls for a more refined model of granular jet entering water to thoroughly understand the dynamics of the jet and the dispersion mechanism of the initially dry grains.

\section{Conclusion}

In this paper, we have investigated the impregnation in a $2 \mathrm{D}$ porous medium plunged from the air into water using experimental, analytical, and numerical approaches. Our results indicate that the impregnation of the grains is characterized by a transient phase followed by a stationary regime. During the transient phase, the shape of the impregnation front continuously evolves to finally reach a stationary V-shape. The impregnation dynamics can be modeled through a 1D impregnation process. Then, the shape of the stationary impregnation front has been experimentally measured and compared to an asymptotic model. This model, based on the Forchheimer's equation, captures well the shape of the impregnation front near the surface of the liquid bath. The front profile is also characterized experimentally for porous media made of wetting and non-wetting grains. We have reported the evolution of the opening angle $\theta$ of the impregnation profile for varying plunging velocity. A scaling law, $\tan \theta \propto\left(V_{0} / V^{\star}\right)^{-0.65}$, is reported for different porous media made of wetting grains. However, a transition from 
an exponent -0.65 to -0.5 in this power law at high plunging speeds is observed for porous media made of non-wetting grains.

Using a numerical approach, we have determined the origin of the exponent -0.65 , which results from a combination of hydrostatic pressure-driven flows with capillary and geometrical effects. The capillary forces are dominating at low penetration depth and affect the exponent value at high plunging speed. The geometrical effects are located in the vicinity of the front tip, where the streamlines concentrate and bent. These geometrical effects modify the scaling law's exponent at low plunging speed since the impregnation front is flattened and curved.

These results aiming at getting a better understanding of how dry grains enter into water have been confronted with experiments of confined 2D granular jets falling into a water bath. Similar observations are reported: a stationary impregnation front exhibiting a V-shape. The front shape follows the scaling law obtained for the porous media. We also provided an estimate of the velocity at which the dense jet of grains enters the water bath. This last result confirms that, at first order, we can assimilate a confined dense granular jet to a porous medium during its impregnation. This work constitutes a first milestone to model the complex interactions between the particles, the interface, and the fluid when grains are entering a liquid.

\section{Appendix A. Characterization of the properties of the grains}

\section{A.1. Size distribution and porosity}

To obtain the different batches of grains used in this study, glass beads are preliminarily sieved by using a sieve column installed on a vibrated table to reduce the granulometric dispersion. The size distributions are then determined by an optical analysis. An example of size distribution is reported for the system W1 in figure 23(a).

The porosity $\varepsilon=1-\phi$ of the porous medium is experimentally estimated by measuring the volume $V_{g}$ occupied by a mass $M_{g}$ of beads. The porosity is given by:

$$
\varepsilon=1-\left(\frac{M_{g}}{\rho_{g} V_{g}}\right),
$$

where $\rho_{g}$ is the density of glass. This measurement highly depends on the preparation of the granular packing. Indeed, a granular material is compacting under vibration or cyclic shearing (Knight et al. 1995; Nicolas et al. 2000; Richard et al. 2005; Kiesgen de Richter et al. 2015). This phenomenon is illustrated in figure 23(b), where we report the progressive compaction of a granular packing as a function of the number of taps. Note that a similar behavior is also observed for granular suspensions and was used as a reliable protocol to prepare controlled samples (Jerome et al. 2016). In our case, for each experiment, the granular material is compacted by tapping the sample 20 times to ensure that the final porosity is reached.

\section{A.2. Contact angle of water on glass beads}

To measure the contact angle of water on glass beads, a drop is generated at the outlet of a needle of diameter $1 \mathrm{~mm}$. Once deposited at the water/air interface, the particle stabilizes at the extremity of the drop and exhibits a contact angle $\theta_{c}$, which can be measured as illustrated in figure 24. Pictures of the particle are recorded with a standard camera and a macro-lens (F-105 Macro). This measurement is performed on 20 beads and averaged to obtain a reasonable estimate of the contact angle. Note that other methods could have been used, such as placing a single particle at the surface of a bath of liquid 


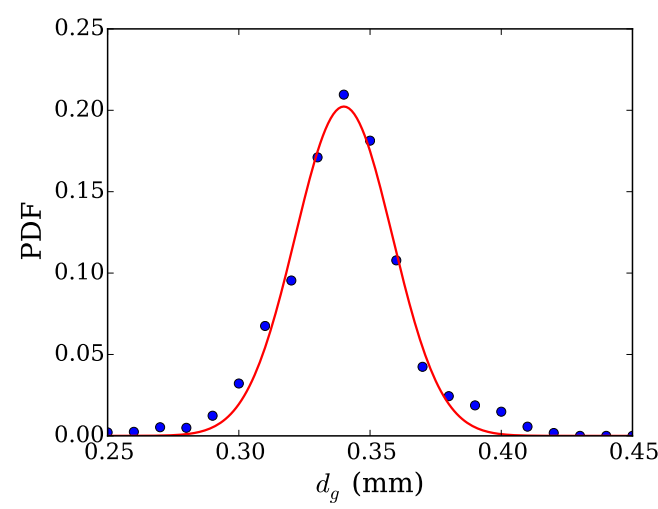

(a)

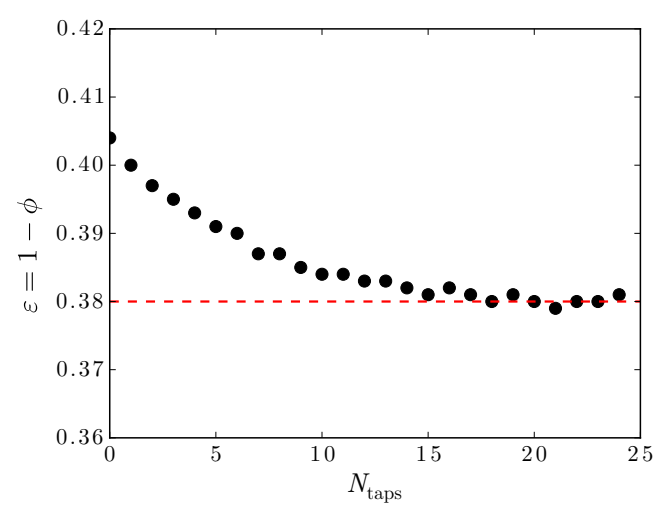

(b)

Figure 23. (a) Example of distribution of the glass beads diameter obtained with the system W1. A gaussian fit is plotted in red line. Here the size of the grains is $d_{g}=340 \pm 40 \mu \mathrm{m}$. (b) Evolution of the porosity $\varepsilon$ of the granular packing during the compaction (Syst. W3). The porosity after compaction is $\varepsilon=0.38$ (horizontal dashed line).

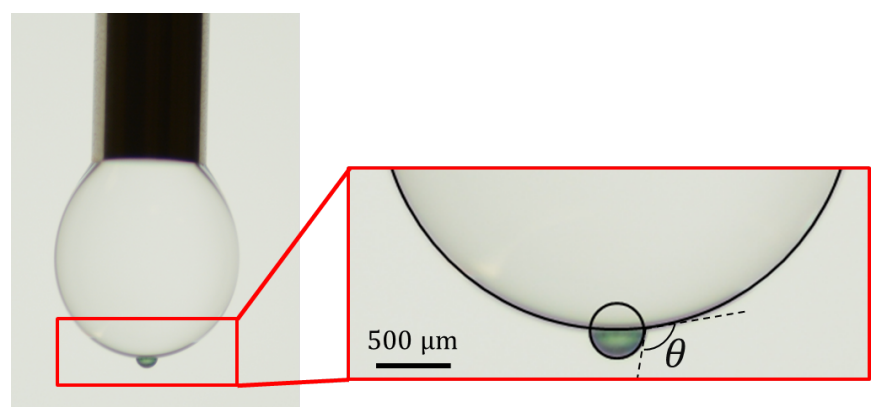

Figure 24. Picture of a glass bead from the system N1 trapped at the surface of a pending drop of water and zoom on the water/air/bead interface. The measured contact angle is $\theta=72^{\circ}$.

and measuring the depth of intrusion of the center of the grain (Raux et al. 2013; Saingier et al. 2017).

\section{A.3. Permeability of the model porous medium}

A custom-made U-shaped tube is filled with glass beads and compacted following the same protocol reported previously. The material is then saturated with water and the two branches of the tube are filled with different level of liquid $z_{1}$ and $z_{2}$ such that $z_{2}>z_{1}$, as shown in figure 25(a). The evolution of the water levels is then recorded and the time evolution of the difference $\Delta z=z_{2}-z_{1}$ is shown in figure $25(\mathrm{~b})$ for the systems W1, W2 and W3. The height $\Delta z$ decreases exponentially with a characteristic time $\tau_{D}$. According to the Darcy's law, the flow velocity $u_{\text {Darcy }}$ through the porous material is:

$$
u_{\text {Darcy }}=-\frac{k}{\eta} \nabla p \quad \text { with } \quad u_{\text {Darcy }}=-\frac{\mathrm{d} z_{1}}{\mathrm{~d} t}=\frac{\mathrm{d} z_{2}}{\mathrm{~d} t} .
$$

The pressure gradient is imposed by the difference of hydrostatic pressure resulting from the difference of altitude $\Delta z$. This leads to the ordinary differential equation describing the time evolution of $\Delta z$ :

$$
\frac{\mathrm{d} \Delta z}{\mathrm{~d} t}=-\frac{2 k}{\eta} \frac{\rho g}{L} \Delta z
$$




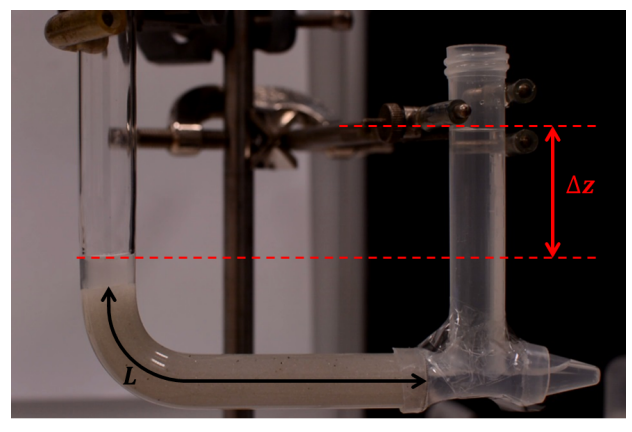

(a)

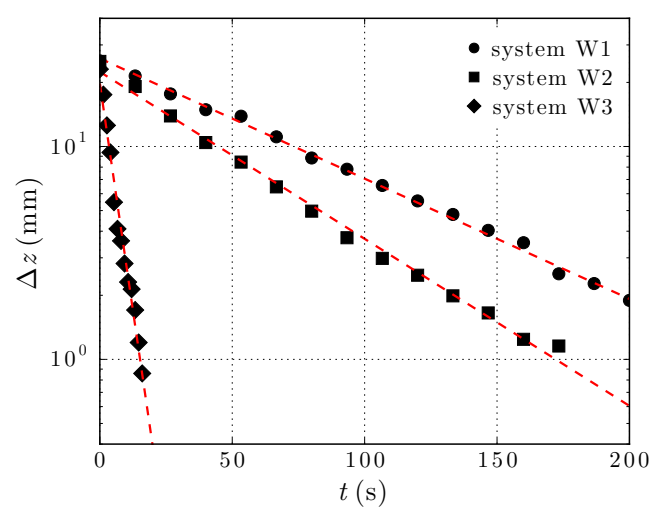

(b)

Figure 25. (a) Picture of the U-shaped tube filled with glass beads over a length $L$. (b) Exponential decrease of the difference of altitude $\Delta z$ as function of time for different systems of glass beads.

where $L$ is the length of the porous medium. The difference of altitude $\Delta z$ decreases exponentially and the characteristic time $\tau_{D}$ depends on the permeability $k$ :

$$
\tau_{D}=\frac{\eta L}{2 k \rho g} .
$$

Measuring the characteristic time of decrease $\tau_{D}$, we obtain the permeability $k_{1}$ reported in table 1.

\section{A.4. Forchheimer coefficient}

The Forchheimer's coefficient $\beta$ is measured by fitting the evolution of the pressure gradient $\nabla p$ as a function of the Darcy's velocity $u_{\text {Darcy }}$ corresponding to the fluid velocity outside of the porous medium. The set-up consisting of the U-shaped tube is modified to impose a larger hydrostatic pressure at the beginning of the experiments. The evolution of the pressure gradient as a function of the Darcy's velocity is shown in figure 26. For small pressure gradients, a linear evolution is observed between $\nabla p$ and the Darcy velocity $u_{\text {Darcy }}$. At larger pressure gradients, we observe a quadratic deviation resulting from the inertial effects. The experimental measurements are fitted by the equation (5.1), and the measurements of the Forcheimer's coefficients $\beta$ are obtained from these fits and summarized in table 1 for the systems W1, W2, and W3.

\section{Appendix B. Analytical expression for the vertical impregnation in a porous medium}

We here derive an implicit solution for the equation describing the impregnation in a $1 \mathrm{D}$ porous medium translating into a water bath at the constant speed $V_{0}$. This situation corresponds to the configuration studied in section 3 . We recall the dimensionless equation (3.7) governing the evolution of $L_{\text {wet }}$ :

$$
\frac{\mathrm{d} L_{w e t}}{\mathrm{~d} T}=\frac{1+\left(V_{0} / V^{\star}\right) T}{L_{w e t}}-1
$$

where $L_{w e t}, T$ and $V^{\star}$ are the dimensionless impregnation length, the dimensionless time and the characteristic speed of impregnation under gravity, respectively, as defined 


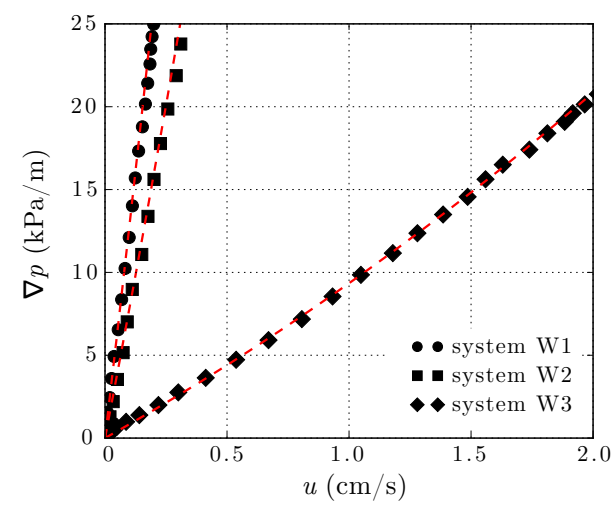

FiguRE 26. Evolution of the gradient pressure as function of the Darcy's velocity for several size distributions and large Reynolds numbers.

in section 3. We introduce a reduced dimensionless time $\tilde{T}=T V_{0} / V^{\star}+1$, and a new variable $\tilde{u}=L_{\text {wet }} / \tilde{T}$ such that the equation (B 1) becomes:

$$
\frac{V_{0}}{V^{\star}} \frac{\mathrm{d} \tilde{u}}{\mathrm{~d} \tilde{T}}=\frac{1}{\tilde{T}}\left(\frac{1}{\tilde{u}}-1-\frac{V_{0}}{V^{\star}} \tilde{u}\right) .
$$

Introducing the parameters $\tilde{U}=\tilde{u}\left(2 V_{0} / V^{\star}\right)$ and $\tilde{V}=\left(4 V_{0} / V^{\star}+1\right)^{1 / 2}$ we obtain:

$$
\frac{\mathrm{d} \tilde{U}}{\mathrm{~d} \tilde{T}}=\frac{1}{\tilde{T}}\left[\frac{\tilde{V}^{2}-(\tilde{U}+1)^{2}}{\tilde{U}}\right] .
$$

Equation (B 3) can be expressed in an integrable form:

$$
\frac{\mathrm{d} \tilde{T}}{\tilde{T}}=\frac{\mathrm{d} \tilde{U}}{2 \tilde{V}}\left[\frac{-(\tilde{V}+1)}{\tilde{V}+\tilde{U}+1}+\frac{\tilde{V}-1}{\tilde{V}-(\tilde{U}+1)}\right]
$$

The solution of equation (B 4), with the initial condition $\tilde{U}(\tilde{T}=1)=0$ corresponding to $L_{w e t}(T=0)=0$, is

$$
\log \tilde{T}_{0}-\log \tilde{T}=\frac{1}{2}\left[\left(1+\frac{1}{\tilde{V}}\right) \log (\tilde{V}+\tilde{U}+1)+\left(1-\frac{1}{\tilde{V}}\right) \log (\tilde{V}-(\tilde{U}+1))\right],
$$

where

$$
\log \tilde{T}_{0}=\frac{1}{2}\left[\left(1+\frac{1}{\tilde{V}}\right) \log (\tilde{V}+1)+\left(1-\frac{1}{\tilde{V}}\right) \log (\tilde{V}-1)\right] .
$$

Finally, the implicit solution can be rewritten as:

$$
\tilde{T}^{2}=\left[\left(1+\frac{\tilde{U}}{\tilde{V}+1}\right)^{1+\frac{1}{\tilde{V}}}\left(1-\frac{\tilde{U}}{\tilde{V}-1}\right)^{1-\frac{1}{\tilde{V}}}\right]^{-1}
$$

At long time, i.e. $\tilde{T} \rightarrow \infty$, we have $1-\tilde{U} /(\tilde{V}-1) \rightarrow 0$, which finally leads to

$$
L_{w e t}=\nu T \quad \text { with } \quad \nu=-\frac{1}{2}+\frac{1}{2} \sqrt{1+4\left(\frac{V_{0}}{V^{\star}}\right)} .
$$




\section{REFERENCES}

Bán, S., Wolfram, E. \& Rohrsetzer, S. 1987 The Condition of Starting of Liquid Imbibition in Powders. Colloids Surf. 22, 291-300.

Bear, J. 1988 Dynamics of Fluids in Porous Media. New-York, USA: Dover.

Benner, E. M. \& Petsev, D. N. 2013 Potential flow in the presence of a sudden expansion: Application to capillary driven transport in porous media. Phys. Rev. E 87 (033008).

Bougouin, A., LaCAze, L. \& Bonometti, T. 2017 Collapse of a neutrally buoyant suspension column: from newtonian to apparent non-newtonian flow regimes. J. Fluid Mech. 826, 918-941.

Campos, J. \& De Carvalho, J. 1992 Drag force on the particles at the upstream end of a packed bed and the stability of the roof of bubbles in fluidised beds. Chemical engineering science 47 (15-16), 4057-4062.

Carman, P. C. 1937 Fluid flow through granular beds. Trans. Inst. Chem. Eng. 15 (150).

Cazacliu, B. \& Roquet, N. 2009 Concrete mixing kinetics by means of power measurement. Cem. Concr. Res. 39 (3), 182-194.

Cervantes-Álvarez, AM, Escobar-Ortega, Yy, Sauret, A \& Pacheco-VÁzquez, F 2020 Air entrainment and granular bubbles generated by a jet of grains entering water. Journal of Colloid and Interface Science .

Cheng, X., Varas, G., Citron, D., Jaeger, H. M. \& Nagel, S. R. 2007 Collective Behavior in a Granular Jet: Emergence of a Liquid with Zero Surface Tension. Phys. Rev. Lett. 99 (18), 188001.

Chopin, J. \& Kudrolli, A. 2011 Building Designed Granular Towers One Drop at a Time. Phys. Rev. Lett. 107, 208304.

Clarke, A. 2002 Coating on a rough surface. AIChE Journal 48 (10).

Collet, R., Oulahna, D., De Ryck, A., Henri, P. \& Martin, M. 2010 Mixing of a wet granular medium : Effect of the particle size, the liquid and the granular compacity on the intensity consumption. Chem. Eng. J. 164 (2-3), 299-304.

Delker, T., Pengra, D. B. \& Wong, P. z. 1996 Interface pinning and the dynamics of capillary rise in porous media. Phys. Rev. Lett. 76 (16), 2902-2905.

Doppler, D., Gondret, P., Loiseleux, T., Meyer, S. \& Rabaud, M. 2007 Relaxation dynamics of water-immersed granular avalanches. J. Fluid Mech. 577, 161-181.

Forny, L., Marabi, A. \& Palzer, S. 2011 Wetting, disintegration and dissolution of agglomerated water soluble powders. Powder Technol. 206 (1-2), 72-78.

Fritz, H. M., Hager, W. H. \& Minor, H. 2003 Landslide generated impulse waves . 1. Instantaneous flow fields. Exp. Fluids 35, 505-519.

González Gutiérrez, J., Carrillo Estrada, J. L. \& Ruiz Suárez, J. C. 2014 Penetration of granular projectiles into a water target. Sci. Rep. 4, 6762 .

Guérin, A., Devauchelle, O. \& Lajeunesse, E. 2014 Response of a laboratory aquifer to rainfall. J. Fluid Mech. 759, 1-11.

Heller, V., Hager, W. H. \& Minor, H. 2008 Scale effects in subaerial landslide generated impulse waves. Exp. Fluids 44, 691-703.

Herminghaus, S. 2005 Dynamics of wet granular matter. Adv. Phys. 54 (3), 221-261.

Horton, R. E. 1945 Erosional development of streams and their drainage basins; hydrophysical approach to quantitative morphology. Geol. Soc. Am. Bull. 56 (3), 275-370.

Hyväluoma, J., Raiskinmäki, P., Jäsberg, A., Koponen, A., Kataja, M. \& Timonen, J. 2006 Simulation of liquid penetration in paper. Phys. Rev. E 73, 036705.

Janssen, H. A. 1895 Versuche uber getreidedruck in silozellen. Z. Ver. Dtsch. Ing. 39 (35), $1045-1049$.

Jerome, J. J. S., Vandenberghe, N. \& Forterre, Y. 2016 Unifying Impacts in Granular Matter from Quicksand to Cornstarch. Phys. Rev. Lett. 117, 098003.

Kirchner, J. W., Feng, X. \& Neal, C. 2000 Fractal stream chemistry and its implications for contaminant transport in catchments. Nature 403 (6769), 524-527.

Knight, J. B., Fandrich, C. G., Lau, C. N., Jaeger, H. M. \& Nagel, S. R. 1995 Density relaxation in a vibrated granular material. Phys. Rev. E 51, 3957-3963.

Lago, M. \& Araujo, M. 2001 Capillary Rise in Porous Media. J. Colloid Interface Sci. 234 (1), $35-43$. 
Lorenceau, E., Restagno, F. \& Quéré, D. 2003 Fracture of a viscous liquid. Phys. Rev. Lett. 90, 184501.

LuCAS, V. R. 1918 Ueber das zeitgesetz des kapillaren aufstiegs von ussigkeiten. Kolloid Z. 23, 15.

Lyle, S., Huppert, H. E., Hallworth, M. A., Bickle, M. \& Chadwick, A. 2005 Axisymmetric gravity currents in a porous medium. J. Fluid Mech. 543, 293-302.

Mensire, R., Ault, J. T., Lorenceau, E. \& Stone, H. A. 2016 Point-source imbibition into dry aqueous foams. Europhys. Lett. 113 (7), 44002.

Müller, P., Formella, A. \& Pöschel, T. 2014 Granular jet impact: probing the ideal fluid description. J. Fluid Mech. 751, 601-626.

Mulligan, R. P. \& TAKe, W. A. 2017 On the transfer of momentum from a granular landslide to a water wave. Coast. Eng. 125, 16-22.

Mullins, B. J. \& BRAdDock, R. D. 2012 Capillary rise in porous, fibrous media during liquid immersion. International Journal of Heat and Mass Transfer 55 (21-22), 6222-6230.

Nasto, A., Regli, M., Brun, P.-T., Alvarado, J., Clanet, C. \& Hosoi, A. E. 2016 Air entrainment in hairy surfaces. Phys. Rev. Fluids 1, 033905.

Nicolas, M., Duru, P. \& Pouliquen, O. 2000 Compaction of a granular material under cyclic shear. Eur. Phys. J. E 3 (4), 309-314.

Courrech du Pont, S., Gondret, P., Perrin, B. \& Rabaud, M. 2003 Granular avalanches in fluids. Phys. Rev. Lett. 90, 044301.

Quéré, D. 1999 Fluid coating on a fiber. Annu. Rev. Fluid Mech. 31 (1), 347-384.

Raux, P. S., Cockenpot, H., Ramaioli, M., Quéré, D. \& Clanet, C. 2013 Wicking in a powder. Langmuir 29 (11), 3636-44.

Reyssat, M., Sangne, L. Y., van Nierop, E. A. \& Stone, H. A. 2009 Imbibition in layered systems of packed beads. Europhys. Lett. 86 (5), 56002.

Richard, P., Nicodemi, M., Delannay, R., Ribière, R. \& Bideau, D. 2005 Slow relaxation and compaction of granular systems. Nat. Mat. 4, 121-128.

Kiesgen de Richter, S., Hanotin, C., Marchal, P., Leclerc, S., Demeurie, F. \& Louvet, N. 2015 Vibration-induced compaction of granular suspensions. Eur. Phys. J. E 38 (7), 74.

Saingier, G., Sauret, A. \& Jop, P. 2017 Accretion Dynamics on Wet Granular Materials. Phys. Rev. Lett. 118, 208001.

Seiwert, J., Clanet, C. \& Quéré, D. 2011 Coating of a textured solid. J. Fluid Mech. 699, $55-63$.

Shirtcliffe, N. J., Mchale, G., Newton, M. I., Pyatt, F. B. \& Doerr, S. H. 2006 Critical Conditions for the Wetting of soils. Appl. Phys. Lett. 89, 094101.

ShojaAee, Z., Brendel, L., Török, J. \& Wolf, D. E. 2012 Shear flow of dense granular materials near smooth walls. ii. block formation and suppression of slip by rolling friction. Physical Review E 86 (1), 011302.

Timounay, Y., Lorenceau, E. \& Rouyer, F. 2015 Opening and retraction of particulate soap films. EPL 111 (2), 26001.

Topin, V., Monerie, Y., Perales, F. \& Radjaï, F. 2012 Collapse dynamics and runout of dense granular materials in a fluid. Phys. Rev. Lett. 109, 188001.

VAnel, L. \& Clément, E. 1999 Pressure screening and fluctuations at the bottom of a granular column. The European Physical Journal B-Condensed Matter and Complex Systems 11 (3), 525-533.

Vella, D. \& Huppert, H. E. 2006 Gravity currents in a porous medium at an inclined plane. J. Fluid Mech. 555, 353-362.

Viroulet, S., Sauret, A. \& Kimmoun, O. 2014 Tsunami generated by a granular collapse down a rough inclined plane. Europhys. Lett. 105 (34004).

Viroulet, S., Sauret, A., Kimmoun, O. \& Kharif, C. 2013 Granular collapse into water: toward tsunami landslides. J. Vis. 16 (3), 189-191.

Washburn, E. W. 1921 The Dynamics of Capillary Flow. Phys. Rev. 17 (3), 273-283.

Xiao, J., Stone, H. A. \& Attinger, D. 2012 Source-like solution for radial imbibition into a homogeneous semi-infinite porous medium. Langmuir 28 (9), 4208-12.

Zitti, G., Ancey, C., Postacchini, M. \& Brocchini, M. 2016 Impulse waves generated by 
snow avalanches: Momentum and energy transfer to a water body. J. Geophys. Res. Earth Surf. 121, 2399-2423. 